This item was submitted to Loughborough's Research Repository by the author.

Items in Figshare are protected by copyright, with all rights reserved, unless otherwise indicated.

\title{
A study on the inclusion of forest canopy morphology data in numerical simulations for the purpose of wind resource assessment
}

\section{PLEASE CITE THE PUBLISHED VERSION}

http://dx.doi.org/10.1016/j.jweia.2013.12.011

\section{PUBLISHER}

(c) Elsevier Ltd (Crown copyright)

\section{VERSION}

AM (Accepted Manuscript)

\section{PUBLISHER STATEMENT}

This work is made available according to the conditions of the Creative Commons Attribution-NonCommercialNoDerivatives 4.0 International (CC BY-NC-ND 4.0) licence. Full details of this licence are available at: https://creativecommons.org/licenses/by-nc-nd/4.0/

\section{LICENCE}

CC BY-NC-ND 4.0

\section{REPOSITORY RECORD}

Desmond, Cian, Simon Watson, Sandrine Aubrun, Sergio Avila, Philip Hancock, and Adam Sayer. 2014. "A Study on the Inclusion of Forest Canopy Morphology Data in Numerical Simulations for the Purpose of Wind Resource Assessment". Loughborough University. https://hdl.handle.net/2134/17732. 
This is the final version of the paper published in Journal of Wind Engineering and Industrial

Aerodynamics, Volume 126, March 2014, Pages 24-37

\title{
A study on the inclusion of forest canopy morphology data in numerical simulations for the purpose of wind resource assessment
}

\begin{abstract}
A series of numerical simulations of the flow over a forest stand have been conducted using two different turbulence closure models along with various levels of canopy morphology data. Simulations have been validated against Stereoscopic Particle Image Velocimetry measurements from a wind tunnel study using one hundred architectural model trees, the porosities of which have been assessed using a photographic technique.

It has been found that an accurate assessment of the porosity of the canopy, and specifically the variability with height, improves simulation quality regardless of the turbulence closure model used or the level of canopy geometry included. The observed flow field and recovery of the wake is in line with characteristic canopy flows published in the literature and it was found that the Shear Stress Transport turbulence model was best able to capture this detail numerically.
\end{abstract}

\section{Introduction}

The numerical theory describing flow through forest canopies has been developed over a number of years. This process has been driven by a need for robust wind resource assessment and also for agricultural applications such as modelling wind loads on isolated forest stands and optimisation of the spraying of pesticides.

A wide variety of Computational Fluid Dynamics (CFD) approaches have been employed with, for example, Yang el al. (2006) favouring the use of Large Eddy Simulation (LES) in order to fully appreciate the turbulent structures which develop across forest edges. LES simulations were also used by Dupont et al. (2010) to investigate the importance of transient effects such as the waving of leaves and branches.

However, for most practical applications, it has been suggested that simpler two equation turbulence models suffice when considering canopy flows (Belcher et al., 2012). This is due to the fact that, although highly turbulent, the flows in these regions are not dominated by the effects of viscosity. This matter will be given further consideration in Section 2.6.

There has been considerable activity investigating how best to implement the effect of canopies in two equation turbulence models and a state of the art has emerged following contributions by Svensson and Haggkvist (1990), Liu et al. (1998), Sanz (2003) and Lopes da Costa (2007). More recently, these two equation canopy models have been modified to include buoyancy effects due to atmosphere stability (Sogachecv et al., 2012) and so it is likely that they will continue to be of use to industry for some time to come. 
In addition, the ability to assess the structure of canopies has also progressed. From the collecting and counting of leaves to the use of various photographic and high density Light Detection And Ranging (LiDAR) techniques, a review of these developments can be found in Jonckheere et al. (2004) and Seidel et al. (2012). In Omasa et al. (2006), a high level of canopy structural detail was acquired using a method which combined GPS, airborne and terrestrial LiDAR as shown in Fig. 1. These data were then combined with temperature and chlorophyll fluorescence measurements in order to allow detailed modelling of the plant physiology.

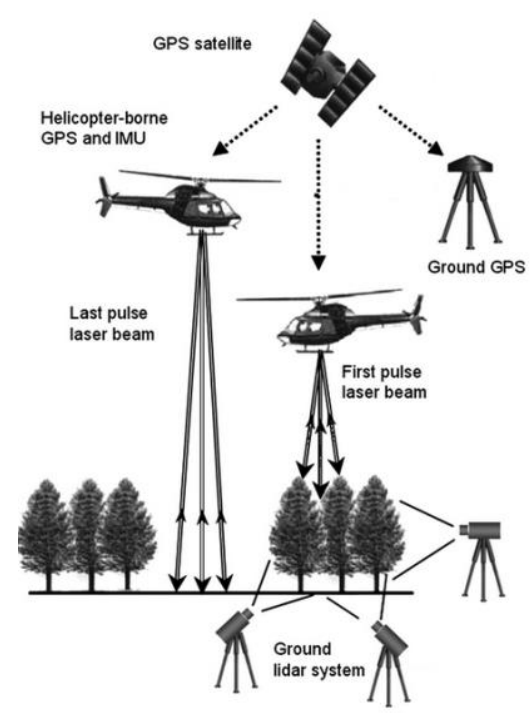

Fig. 1. Capturing canopy structural data. From Omasa et al. (2006).

Various authors have suggested that these parallel advances in numerical theory and the ability to capture morphology data could be used in combination to reduce the uncertainty associated with modelling canopy flows.

In Endalew et al. (2009a) it was noted that much work has been carried out in accurately capturing the structural detail of forest canopies, but little effort had been expended to implement this in CFD modelling. These authors used an extremely high level of canopy detail to conduct CFD modelling of flow around a pair of model trees using LES. Individual branches were explicitly modelled and the effect of the leaves was introduced within tight fitting porous sub-domains around each branch. Simulations were validated using wind tunnel data and it was found that this high level of canopy detail improved the quality of the CFD results albeit at a considerable computational expense.

More recently, Burns et al. (2011) pointed to the importance of canopy morphology based on investigations of data from five forested sites and suggested that the required structural data would be best captured using high density LiDAR systems. In Dupont et al. (2012), a number of LES simulations were conducted based on forested site data and in Lee and Lee (2012), the flow around a bank of real fir trees was investigated by use of Stereoscopic Particle Image Velocimetry (stereoPIV) in a wind tunnel. Again, both Dupont et al. (2012) and Lee and Lee (2012) concluded that an understanding of the actual morphology is vital when modelling canopy flows.

Whilst a body of opinion clearly exists that CFD simulations will benefit from the incorporation of morphology data, it is important to note that the work mentioned above is concerned with aspects of canopy flow such as wind loading on individual trees and turbulent structures within canopies. 
Whilst of interest, these factors may not be directly applicable to the concerns of the wind energy industry. In this paper, we will focus on elements of the flow above and around canopies of direct relevance to the resource assessment community. These are the modulus of the mean wind speed, $T^{\prime \prime} X$, and mean Turbulent Kinetic Energy $(k)$.

In order to provide an extensive validation database, experiments have been conducted in an atmospheric boundary layer wind tunnel to investigate the wake around a stand of 100 architectural model trees. Subsequently, canopy morphology data were captured using photographic analysis software and a series of CFD simulations were conducted in which increasing levels of detail were gradually introduced. By analysing the quality of these simulations, we have investigated whether a detailed understanding of the canopy structure is beneficial when modelling flow around a forest canopy for the purpose of wind resource assessment.

\section{Experimental data}

\subsection{Tunnel description}

Experiments were conducted in the Lucien Malavard wind tunnel which is located in the Laboratoire Prisme, University of Orléans, France. As can be seen from Fig. 2, this is a close-return wind tunnel in which two sections are available for testing. Experiments for this study were conducted in the $5 \mathrm{~m}$ wide $\times 5 \mathrm{~m}$ high $\times 12 \mathrm{~m}$ long secondary test section which utilises a turbulence grid, turbulence spires and a rough metallic floor plate to generate a scaled atmospheric boundary layer to a maximum wind speed of $10 \mathrm{~m} / \mathrm{s}$.

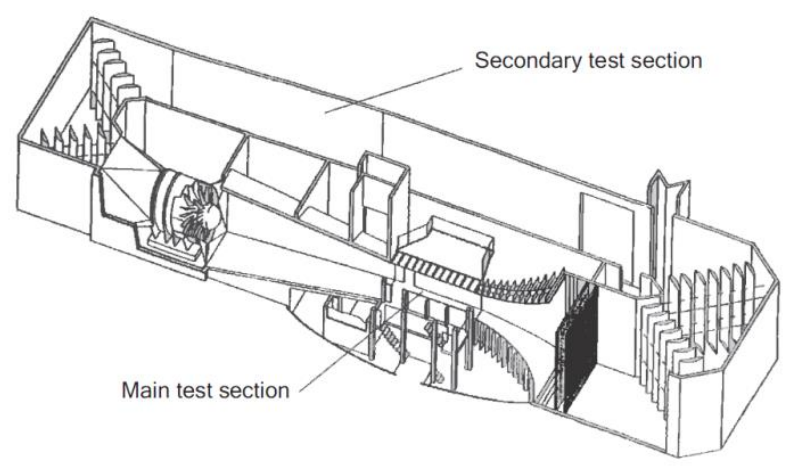

Fig. 2. Schematic view of the wind tunnel in University of Orléans.

Measurements were performed using stereo-PIV (Schröder and Willert, 2008). The flow was seeded with a fine mist of olive oil ( $1 \mu \mathrm{m}$ i $\mathrm{n}$. The ade moe itterest ) was illuminated by use of an NdYAG twin laser (model CFR PIV 190, manufactured by Big Sky Laser, $200 \mathrm{~mJ} /$ pulse) to generate a laser light sheet. In order to acquire the required images, two POWERVIEW Plus $4 \mathrm{M}$ cameras (model $630059,2048 \times 2048$ pixel resolution) were positioned to capture a stereoscopic view of the illuminated flow field. Both cameras and lasers were synchronised by use of a LASERPULSE module (model 610035) with a sampling frequency of $7 \mathrm{~Hz}$, the maximum allowable by the equipment. Signal acquisition and processing of raw data were then performed using Insight software, provided by TSI, to produce a three dimensional flow field for the plane under investigation.

The setting of the stereo-PIV was performed by using the TSI Dual Plane/Dual Sided calibration target. A total of 2000 images were captured for each of a series of concatenated $360 \mathrm{~mm}$ wide $x$ $350 \mathrm{~mm}$ high measurement planes. An overlap of $60 \mathrm{~mm}$ was used between planes in order to improve the merging of data through linear interpolation. 
An adaptive interrogation window was used, starting from $64 \times 64$ pixels to a final $32 \times 32$ pixels window. The interrogation window overlap was $25 \%$ and the analysis was based on FFT Correlation algorithm and the Gaussian peak fitting technique. Analysis of these data allowed both mean and variances to be deduced for the three main instantaneous wind speed components $U, V, W$. In order to automate the measurement process, both the cameras and laser equipment were placed on an ISEL traverse system. The configuration used in the tunnel can be seen in Fig.3.

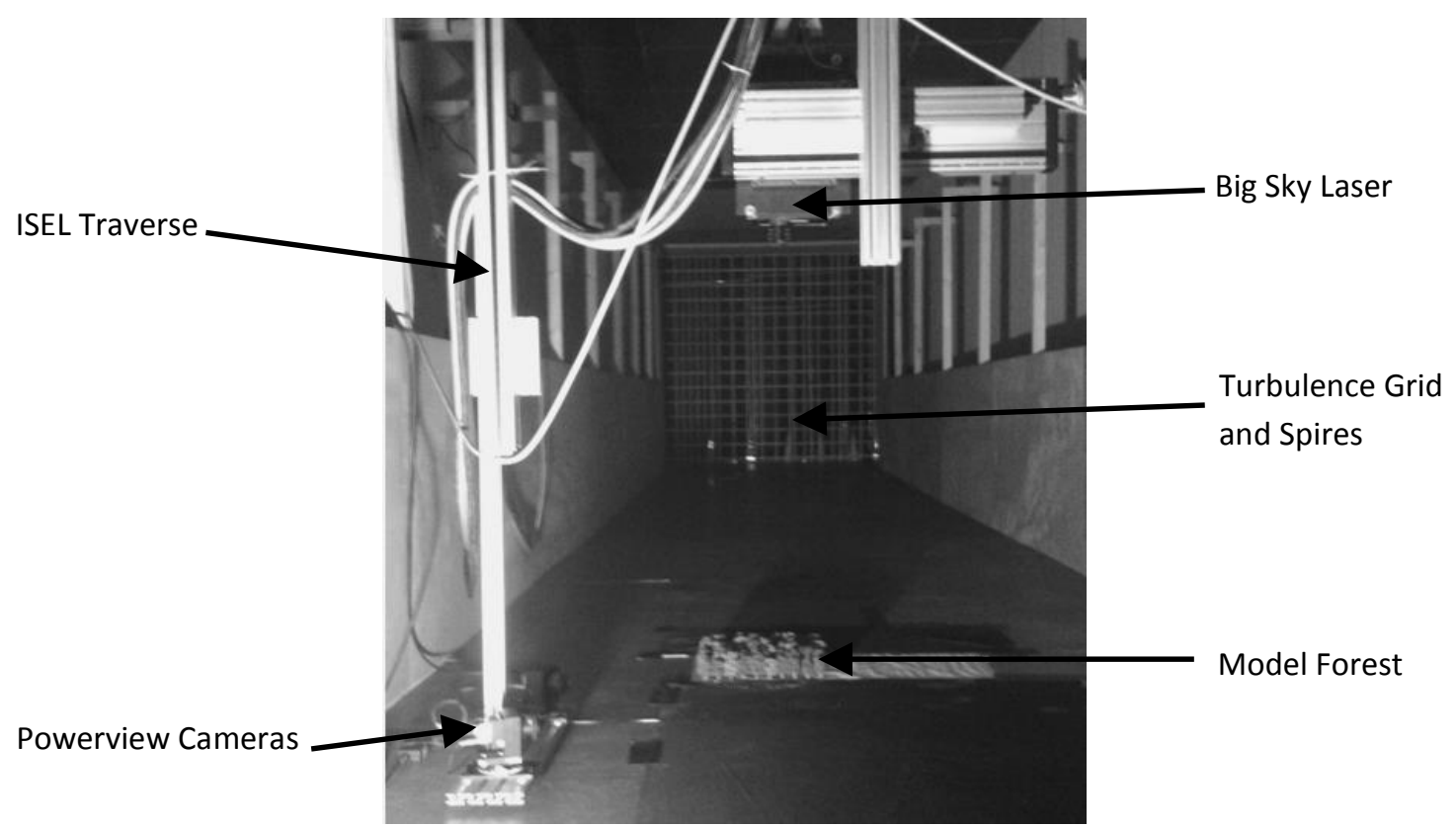

Fig. 3. View upstream in the Lucien Malavard wind tunnel.

It is difficult to quantify the experimental error associated with the stereo-PIV measurement technique. A discussion on potential error sources and a suggested uncertainty analysis methodology can be found in Zhang et al., (2003). However, in this paper the data produced by the stereo-P I V s y s t e m wi l be $t$ a k e $n$ a $t h e$ " $r$ u e val ues"

\subsection{Boundary layer simulation}

A boundary layer representative of moderately rough open terrain with low vegetation in neutral stability was simulated at a scale of 1:300 during the course of these experiments in accordance with VDI - guideline $3783 / 12$ (2000). The vertical profile of the fully developed inlet wind speed is described by the power law:

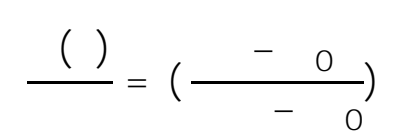

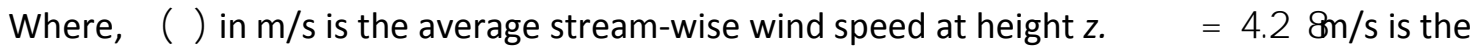

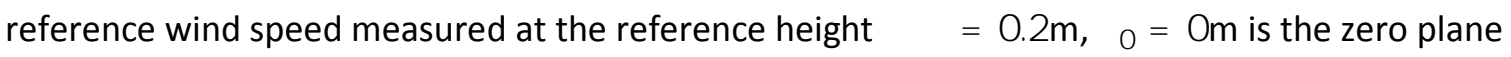
displacement height and $5,\llcorner \& \xi$ is the shear exponent. Close to the ground, the velocity is approximated by:

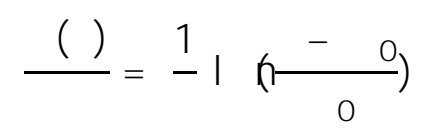




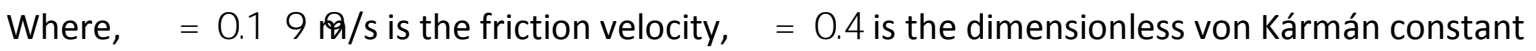

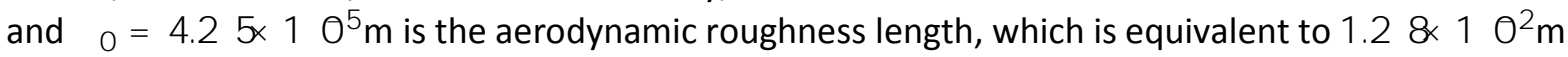
at full scale. Graphs of the experimental windspeed and Turbulent Kinetic Energy profiles are given in Section 3.3 where they are compared with the inlet profiles used for the CFD simulations.

\subsection{Model trees}

A wide variety of materials have been used to simulate the effect of forest canopies in wind tunnel studies. These include blocks of foam rubber (Rodrigo et al., 2007), Lego ${ }^{\mathrm{TM}}$ bricks , Meccano ${ }^{\mathrm{TM}}$ wheels (Stacy et al., 1994), wooden pegs, wire mesh (Aubrun and Leitl, 2004), miniature fir trees (Lee and Lee, 2012), bottle brushes (Liang et al., 2005), 3D printed fractal models (Bai et al., 2012), automotive bulbs (Böhm et al., 2013) and architectural model trees (Meroney, 1968, Endalew et al., $2009 \mathrm{~b}$ and Ruck et al., 2012). Each of these materials have their own merits and allow various aspects of canopy flows to be investigated faster, cheaper and in greater detail than a full-scale experimental campaign.

In order to validate the CFD simulations against these experiments, it is necessary to quantify the canopy structure in some way. This is often achieved by tuning the CFD to identify the correct porosity for the experimental model, however, other options exist. In the case of Liang et al. (2005), the shadow of an individual brush was examined as a proxy for the vertical structure of the tree canopy. In Bai et al. (2012), the actual geometry file used for the 3D printing was utilised whilst in Endalew et al. (2009 b) and Lee and Lee (2012), photographic techniques were used to capture the required canopy structural data.

In this paper, we have chosen to use architectural replica trees similar to those used by Endalew et al. (2009b). These models, which were sourced from the 4D Modelshop Ltd. (London, UK), are composed of etched brass wire with rough ground silicon to simulate foliage, Fig. 4. These model trees allow a high level of structural data to be captured using photographic techniques, which will be discussed in Section 2.5, whilst also allowing for repeatability of the experiments that could not be achieved using live specimens.

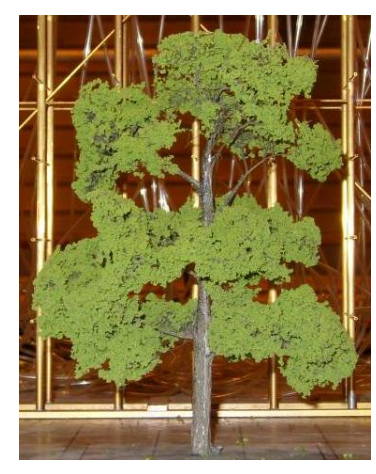

Fig. 4. Architectural model tree of the type used during the experiments.

However, these model trees have a number of limitations. For instance, the artificial leaves are stationary and are thus unable to interact with the wind, which may be an important factor in forest flows (Finnigan, 2010). Similarly, the ridged branches are unable to streamline to the incident flow 
which results in a coefficient of drag which is independent of the magnitude of the incident wind speed which is not the case in actual canopies (Molina-Aiz et al. 2006).

Also, as discussed in Böhm et al. (2013), the use of scale models of vegetation in wind tunnels may result in the flow being dominated by viscous drag which will not be representative of full-scale flows. However, the use of non-bluff bodies to represent forest canopies in wind tunnels is not without precedent (Meroney, 1968, Liang et al., 2005, Endalew et al., 2009 b, Lee and Lee, 2012 and Ruck et al., 2012). These limitations aside and given that the focus of this study is the role that detailed canopy morphology can play in improving simulations, these model trees were deemed to be the best candidate for experimentation.

\subsection{The forest set-up}

The miniature forest used in the Orléans experiments was comprised of 100 architectural model trees of the type discussed in Section 2.3. A range of tree types were selected to ensure that the vertical structure of the canopy was strongly heterogeneous, as would be expected for a mixed forest containing a variety of tree species. In addition, advice was taken (Dr. Jonathan Millet, Geography dept, Loughborough University, personal communication, May 1, 2012) as to the range of heights that would be expected in a mixed forest. The distribution used is shown in Fig. 5 where the mean canopy height $H_{c}=76 \mathrm{~mm}$.

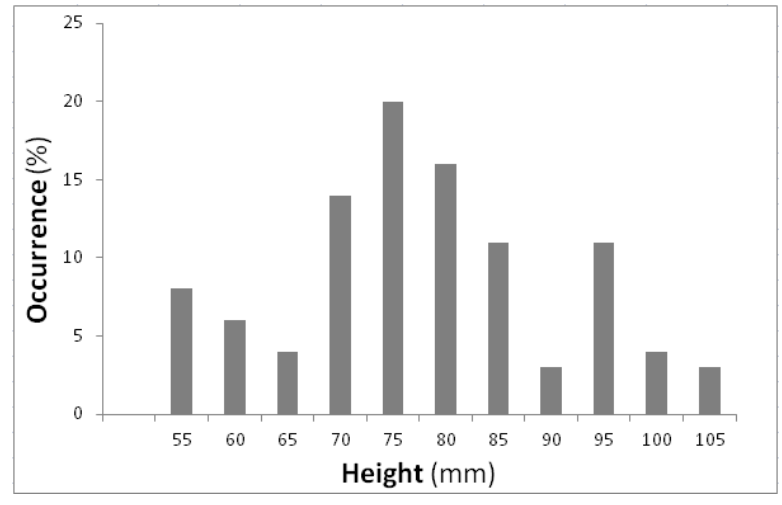

Fig. 5. Height distribution of the model forest.

B a s ed on a uthor's own measurement s.

Within the tunnel, the trees were placed randomly with an approximately equal spacing in an area of $675 \mathrm{~mm} \times 675 \mathrm{~mm}$. The placement of the trees was carefully recorded in order to allow accurate replication of the experiment using the CFD code. These various steps ensured that the canopy was heterogeneous in order to allow realistic 3D flow patterns to develop.

Given the relatively small footprint of the forest, approximately $10 \times 10$ trees, it is possible that the flow will not be fully adjusted to the increased roughness that it introduces. In Belcher et al., (2003) and elsewhere, the canopy adjustment length $L_{c}$ is calculated as:

$$
\text { ०० } \kappa x\left(\text { सP }_{\mathrm{P}}\right. \text {, ष । प्रो ग़ }
$$

Where, Bulk $=8.34 \mathrm{~m}^{-1}$ is an averaged value of the Leaf Area Density which describes the bulk structure of the canopy as shown in Fig. 6 . Using a value for the drag coefficient $C_{d}=0.25$, as 
recommended in Endalew et al. (2009b) for similar model trees, $L_{c}$ is calculated as $479 \mathrm{~mm}$. This would indicate that, as the total canopy length is $675 \mathrm{~mm}$, the flow over the model forest will be fully adjusted to the canopy roughness.

The average spacing between the trees is $0.95 H_{c}$ and the plan roughness density $\bar{\Sigma}_{\Omega_{-}} \mathrm{I}$ श $_{\Omega} \mathrm{E}$ श $=$ 0.341 , where $\mathrm{I}_{\mathrm{I}} \mathrm{T}_{\Omega}$ is the sum of the maximum plan area of each tree and $A$ is the total plan area covered by the forest. These values are characteristic of a sparse canopy and so there will be some residual drag effect from the underlying roughness of the wind tunnel floor.

\subsection{Determining the morphology}

In this study, canopy morphology is taken to be comprised of LAD data and also geometry data such as the canopy height, crown diameter and location of individual trees. These geometry data have been obtained by recording the placement of each tree within the forest and by taking detailed measurements of each individual tree using a digital calliper. The required LAD data were acquired using the method discussed below.

The LAD is a convenient metric for describing canopy density which is commonly used in the forestry community. It is calculated within a horizontal section through the forest at some height and is defined as the total one-sided leaf area within that section divided by its volume. By taking a series of horizontal sections at a number of different heights, a height-varying LAD profile can be defined which gives an appreciation of the vertical canopy structure.

As discussed in the introduction, there are a number of different methods of determining this LAD profile for forest canopies. In this study, we have used a method which required eight photographs to be taken of each model tree using a digital camera. These images were then converted into black and white bitmaps which were used by the software Tree Analyser (Phattaralerphong and Sinoquet, 2005) to compute the LAD profile.

The finest accuracy settings were used for the analysis which resulted in each profile taking approximately six hours to produce using the Tree Analyser software. This was in order that the results were of a sufficient level of detail to be analogous to a full LiDAR survey of an actual canopy where a resolution of $30 \mathrm{~mm}$ is recommended (Seidel et al., 2012). A full explanation of the calculations performed by the software can be found in Phattaralerphong and Sinoquet (2005).

As Tree Analyser is designed for use on full scale canopies, it was necessary to make modifications to analyse the scale models. These adjustments did not cause a problem when analysing larger $175 \mathrm{~mm}$ trees (Endalew et al. 2009b and Desmond and Watson, 2011), however it was noticed that the LAD profile was overestimated for smaller $80 \mathrm{~mm}$ trees (Desmond and Watson, 2012).

In order to investigate the extent of this discrepancy, data from a series of wind tunnel experiments examining the flow around single $80 \mathrm{~mm}$ conifers of various porosities were used for CFD validation. The magnitude of the LAD profile as calculated using Tree Analyser was gradually reduced in a series of CFD simulations in order to identify the appropriate reduction. This value was found to be $75 \%$ regardless of the porosity of the model canopy under investigation. This reduction was applied to all profiles calculated for the 100 model trees which comprised the miniature forest used in the Orléans experiments. The resulting adjusted average profile for the entire canopy is shown in Fig. 6 . 
It is possible that the overestimation of the effect of the canopy using the LAD profiles determined using Tree Analyser is in part due to the value of $C_{d}$ used. This value was determined in Endalew et al., (2009b) for $175 \mathrm{~mm}$ model trees and a reduced value may be more appropriate for the smaller models. It is unclear whether an adjustment should be made to the LAD profile values, the value of $C_{d}$ or a combination of both for the smaller scale trees. However, as the product of $C_{d}$ and LAD is always used in the CFD calculations, Eqs. 9-12, the effect of reducing the LAD alone is sufficient.

The LAD profile which describes the vertical structure of the miniature forest is shown in Fig. 6 in which $H_{c}=0.076 \mathrm{~m}$ is the average canopy height and $H_{T}=0.019 \mathrm{~m}$ is the average trunk height. Bulk = $8.34 \mathrm{~m}^{-1}$ is the average of the LAD profile between these limits as per Andersen et al., (2005). The average value for the entire profile shown in Fig. 6 is $L A D=5.75 \mathrm{~m}^{-1}$. Dimensions have been normalised to $H_{c}$ in order to facilitate comparison with other graphs in this paper.

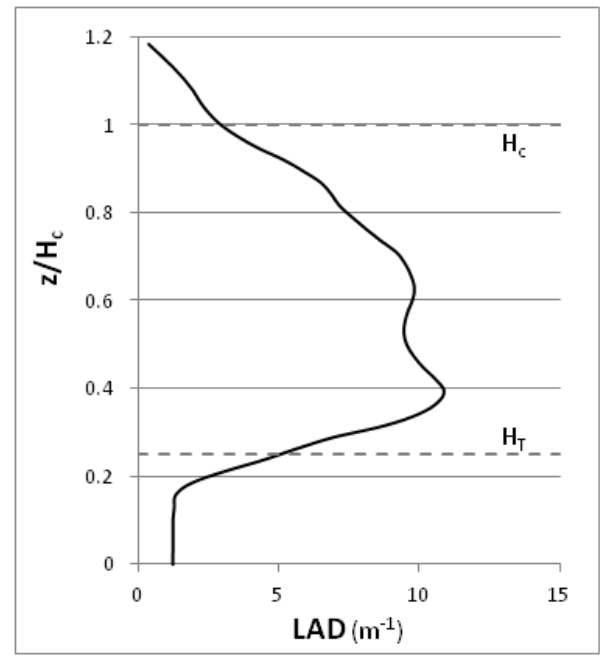

Fig. 6. LAD profile for the entire forest canopy.

The Bulk LAD is the average of this profile between $\mathrm{H}_{c}$ and $\mathrm{H}_{T}$

\subsection{Reynolds number dependence}

The dimensionless Reynolds number, $R e$, which relates the relative importance of viscous and inertial forces, is an important consideration when assessing the relevance of wind tunnel experiments, particularly given the concerns raised in Böhm et al. (2013) relating to the use of nonbluff bodies to represent forest canopies at wind tunnel scale. This quantity is defined as:

$$
-\frac{\text { ज़ॉ" थ }}{3}
$$

Where, $l$ in $\mathrm{m}$ is the characteristic length scale, 2 in $\mathrm{kg} / \mathrm{m}^{3}$ is the density of air and 3 in $\mathrm{kg} / \mathrm{m} . \mathrm{s}$ is the dynamic viscosity.

The selection of a characteristic length scale when considering flows within the atmospheric boundary layer is a non-trivial task and the value used often varies depending on the preference of the author (Petersen, 2013). Given the complexity of the canopy used in this study, a variety of 
possible values exist from the diameter of an average branch to the height of the boundary layer itself.

Thus, in order to investigate the Reynolds number dependence of the flow around the model trees, an experiment has been conducted at a separate wind tunnel facility.

\subsubsection{Tunnel description}

An experiment investigating the Reynolds number dependence of the flow around the model trees was conducted in the EnFlo atmospheric boundary layer tunnel located at the University of Surrey, UK. This is a twin-fan suck-through facility with an operational section of $1.5 \mathrm{~m}$ high $\times 3.5 \mathrm{~m}$ wide $\times$ $20 \mathrm{~m}$ long. The tunnel is one of the few atmospheric boundary layer facilities available for research purposes that can be thermally stratified; however, it was run using neutral stratification during this experiment.

The flow around a $175 \mathrm{~mm}$ conifer and an $80 \mathrm{~mm}$ conifer of similar LAD was investigated by use of Laser Doppler Anemometry (LDA) for a free-steam wind speed ${ }^{\# "}{ }^{\prime \prime}$ ref $=2.5 \mathrm{~m} / \mathrm{s}$. An aerodynamic roughness length of $z_{0}=3 \times 10^{-5} \mathrm{~m}$ for the tunnel floor was used during the experiment. Due to time limitations, it was not possible to set up rural boundary layers with appropriate scaling for each of the model trees individually.

Measurements were taken to produce a series of mean horizontal wind speed $\mathrm{I}^{\sharp}$ and $k$ profiles at nine distances from $0.5-15 H_{c}$ behind each of the model trees. Results were then normalised to the respective $H_{c}$ in order to investigate the Reynolds number dependence of the flow. Results are presented in Section 2.6.2 for the profiles measure at $2 \mathrm{H}_{c}$ and $5 \mathrm{H}_{c}$ behind each of the model trees. 


\subsubsection{Results}

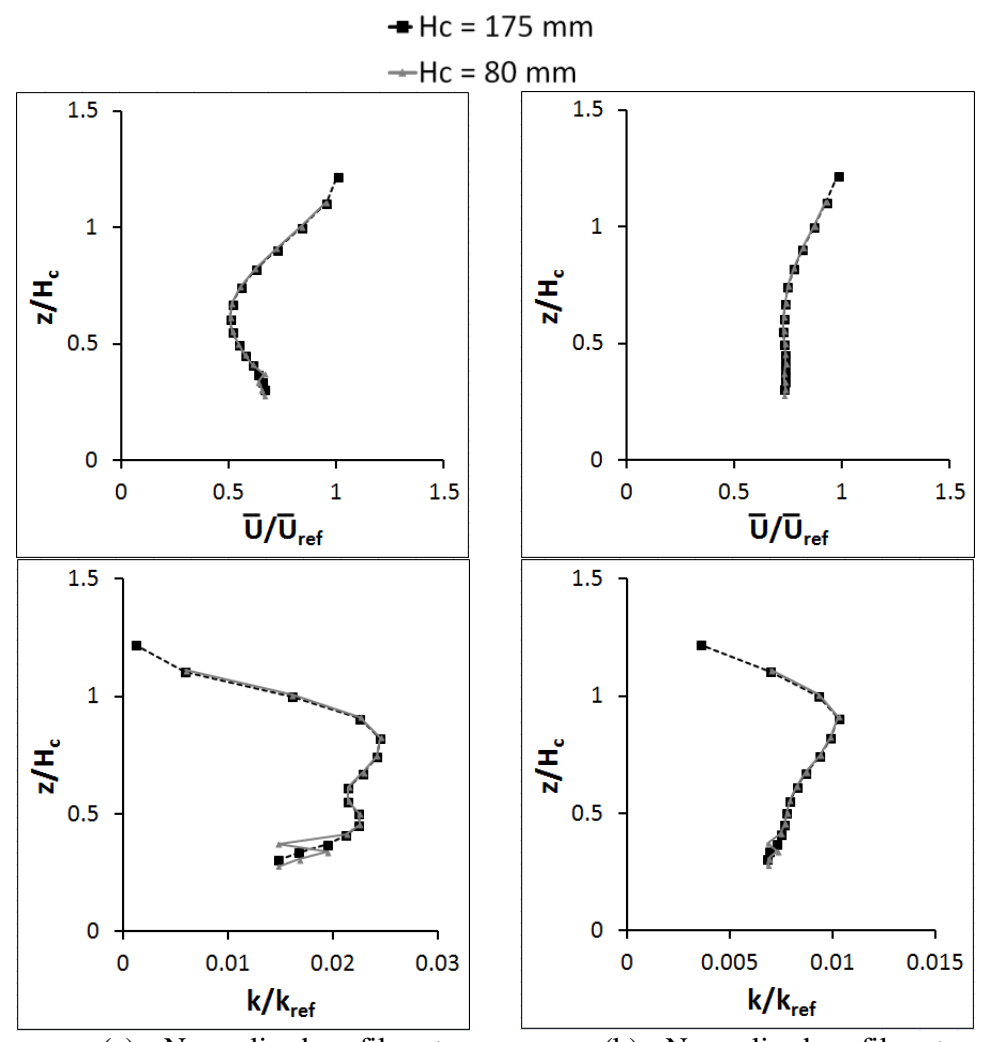

(a) Normalised profiles at $2 H_{C}$.

(b) Normalised profiles at $5 H_{C}$.

Fig. 7. Normalised profiles for $k$ and 药 at $H_{c}$ and $5 H_{c}$ behind trees of $80 \mathrm{~mm}$ and $175 \mathrm{~mm}$.

The normalised velocity profiles in Fig. 7 show a good collapse of the normalised data which would suggest that the flow around the architectural model tress is not heavily dependent on the Reynolds number. This matter will be given further consideration in Section 5 , where we will compare the observed flow field around the miniature forest to data for full-scale canopy flows.

\section{Simulations}

\subsection{Description of the CFD model}

The commercially available CFD software ANSYS CFX 14.0 was used for this study. CFX contains a coupled solver for mass and momentum which allows the Reynolds Averaged Navier Stokes (RANS) forms of the governing equations to be solved for a user defined node-centred grid using an algebraic multi-grid algorithm for convergence acceleration. This is a generalised numerical fluid dynamics solver and requires user modification in order to simulate flows within the atmospheric boundary layer.

The inlet velocity profile for the neutrally stratified boundary layer is described by Eqs. 1 and 2 . The aerodynamic roughness length $z_{\mathrm{o}}$ is converted to the equivalent sand grain roughness length, $\varepsilon_{s}$, using Eq. 5 (McCormick et al., 2012):

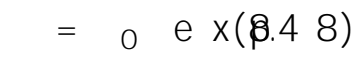


This value is then applied to the floor of the CFD model domain. The Turbulent Kinetic Energy $k$, and its dissipation rate, $\varepsilon$, in a fully developed neutral atmospheric boundary layer are defined by Eqs. 6 and 7 respectively (Richards and Hoxey, 1993):

$$
\begin{aligned}
& \text { T़ } \frac{1^{76}}{\dot{p}^{\overline{स_{\bar{r}}}}}
\end{aligned}
$$

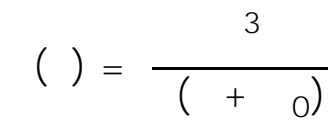

Where $C_{\mu}=0.09$ is a modelling constant. In order to provide a comparison, both the standard $k-\varepsilon$ (Launder and Spalding, 1972) and Shear Stress Transport (SST) (Menter, 1993) turbulence models have been used for closure of the RANS equations in this study.

The limitations associated with using the standard $k-\varepsilon$ model for investigations within the atmospheric boundary layer are well documented (Franke et al., 2007). Some of these limitations can be circumvented by adopting modified forms of the standard $k-\varepsilon$ equations, such as the realizable or RNG models, which along with the standard $k-\varepsilon \quad$ mared popular choice for those conducting simulations for the purpose of resource assessment (Bechmann et al., 2011). As the standard $k-\varepsilon \quad$ mode $\mathrm{I}$ i s $\mathrm{t}$ he mos $\mathrm{t}$ wi del y used t urbulence important reference in the boundary layer meteorology community (Sogachev et al., 2012) it will be used here as a benchmark for the more advanced SST model.

The SST model is a hybrid of the $k-\varepsilon$ athe $k-\bar{r}$ turbulence closures. The $k-\bar{m}$ model was developed by Wilcox (1998) in order to deal with some of the problems associated with the $k-\varepsilon \quad m$ outhen I considering flow close to surface elements due to a singularity in the governing equations. The $k-\bar{s}$ model overcomes this problem by introducing a slight modification whereby the turbulent eddy frequency, $\omega$, is considered rather than the dissipation rate, $\varepsilon$. The equation for Turbulent Eddy Frequency is:

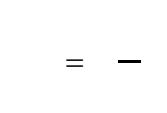

\subsection{CFD simulation of canopy flows}

In order to represent a forest canopy within the CFD model, a porous sub-domain is introduced. By including production terms for Turbulent Kinetic Energy $k$, Turbulent Eddy Frequency, $\omega$, and Turbulent Eddy Dissipation, $\varepsilon$, within this sub-domain, the effect of a forest canopy can be simulated. This is achieved by the addition of source terms in the governing equations for momentum and turbulent transport. In the momentum equation, a drag term is introduced:

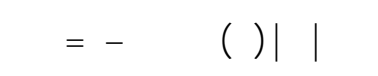


Where, $F_{i}$ in $\mathrm{kg} / \mathrm{m}^{2} . \mathrm{s}^{2}$, is the drag force per unit volume in the i-direction, $A(z)$ in $\mathrm{m}^{-1}$ is the LAD at height z. The term $T$ in $\mathrm{m} / \mathrm{s}$ refers to the modulus of the windspeed and ${ }^{\top}$ oralso in $\mathrm{m} / \mathrm{s}$ is the wind speed in the $i$-direction. The source term for $k$ is:

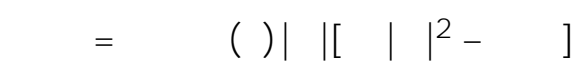

Where, $\nabla_{\Omega}$ and $\nabla_{\rho}$ are constants, the values of which are given in Table 1 . The source term for Turbulent Eddy Dissipation, $\varepsilon$, is given by:

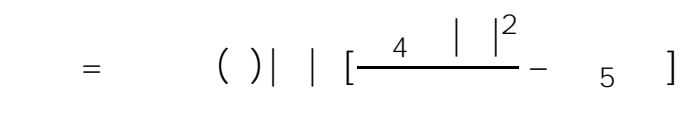

Where, सegrand सesrare constants, the values of which are also given in Table 1 . The source term for Turbulent Eddy Frequency, $\omega$, is given by:

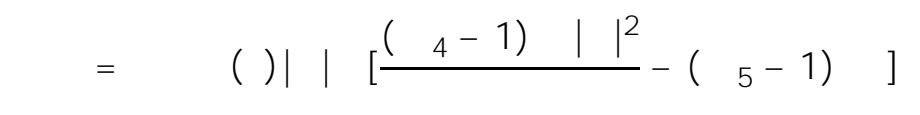

A discussion on the formulation of these equations can be found in Lopes da Costa (2007) and Sogachev (2009).

It will be noted that the source and sink terms for turbulent transport equations, Eqs. 10-12 are themselves functions of $k$, №and ₹. This can cause convergence problems for the solver as a feedback loop will exist between the magnitude of the variable and how it is affected by the canopy. In order to assess the sensitivity of simulations to the values of $k$, №and $₹$ within Eq. 10-12, both inlet and local values have been used.

In order to avoid unjustified tuning of the CFD simulations, prescribed values were employed for all variables and constants. The LAD profile obtained by the method described in Section 2.5 was used along with the value of $C_{d}=0.25$. A range of values have been suggested by various authors for the required modelling constants. In the absence of any prevailing consensus we have opted to use

\begin{tabular}{|c|c|}
\hline Constant & Value \\
\hline सु $_{3}$ & 0.09 \\
\hline$\nabla_{\Omega}$ & 0.17 \\
\hline$\nabla_{\rho}$ & 3.37 \\
\hline सugr $_{\text {or }}$ & 0.9 \\
\hline सer $_{0}$ & 0.9 \\
\hline
\end{tabular}
those recommended in Lopes da Costa (2007) which are summarised in Table 1.

Table 1. Momentum and turbulence equation constants used for simulating the forest canopy. 


\subsection{The CFD model set-up}

The boundary layer within the CFD model was configured to match the fully developed boundary of the wind tunnel which was measured upstream of the forest. The resulting non-normalised profiles are compared in Fig. 8. Due to the high density of data available, profiles are presented as a continuous series. Measurements lower than $20 \mathrm{~mm}$ are not available for the wind tunnel due to problems with reflections from the metallic mesh on the floor.

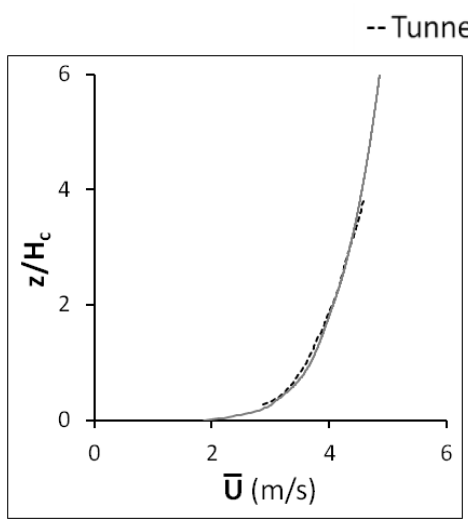

(a) Velocity

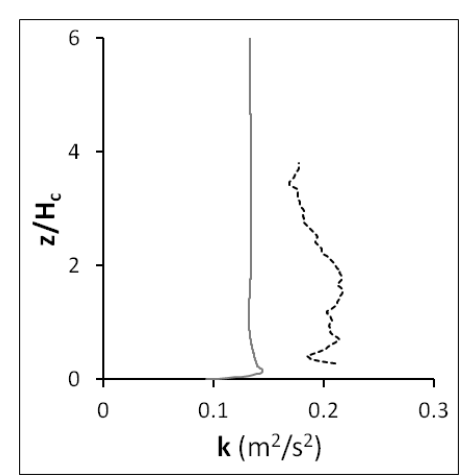

(b) Turbulent kinetic energy

Fig. 8. Inlet profiles from the CFD model and the wind tunnel. Profiles are measured at a distance of $5 \mathrm{H}_{c}$ upstream of the forest in both the CFD and the wind tunnel in order to coincide with the start of the stereo-PIV measurement plane.

As can be seen in Fig. 8, there is good agreement between the CFD model results and the wind tunnel measurements for $\stackrel{\ddot{H}}{ }$, however, the values for $k$ from the CFD model are slightly lower. It is possible to remove this discrepancy by adjusting the value of I used in Eq. 6 to determine the profile within the CFD model. However, this modification causes instability and thus undesirable development of the boundary layer within the domain.

It is also possible to simply use the $k$ profile as measured in the wind tunnel for the relevant boundary conditions in the CFD. However, this was also found to cause excessive problems with horizontal heterogeneity within the domain. A discussion on the implications of such conditions can be found in (Juretić and Kozmar, 2013). As the magnitude of the error is low, approximately 0.08 $\mathrm{m}^{2} / \mathrm{s}^{2}$, the profiles in Fig. 8 were considered satisfactory for all simulations.

The CFD model domain used for this analysis was $5.7 \mathrm{~m}$ long $\times 2.3 \mathrm{~m}$ wide $\times 1 \mathrm{~m}$ high.

A mesh sensitivity study was conducted in which three different unstructured tetrahedral meshes were investigated. Each mesh was refined in two zones. The first, Zone 1, coincided with the volume of the porous sub domain representing the forest. The second, Zone 2, extended to $40 \mathrm{H}_{c}$ behind, $7 \mathrm{H}_{c}$ in front, $4 H_{c}$ above and $3 H_{c}$ to either side of the forest. A constant cell size was used in Zone 1 whilst a geometric growth rate of 2 was used in Zone 2. Details of the maximum element size applied to these zones for each mesh can be found in Table 2. 
For all meshes, a five cell inflation layer of hexahedral elements was defined at the lower boundary of the domain which represented the floor of the tunnel. The first cell was set at $2.4 \mathrm{~mm}$ with a geometric growth rate of 1.2 .

Simulations for both the mesh sensitivity study and the main body of this paper were conducted on the Loughborough University research High Performance Computing (HPC) cluster which consists of 161 nodes, each having two six-core Intel Westmere Xeon X5650 CPUs and 24GB of memory. Each simulation was divided among twelve cores in order to avoid problems which may occur from segmenting the domain into an excessive number of parallel computations.

Descriptions of the three meshes used for the sensitivity study are presented in Table 2 along with the time in minutes required for each simulation to converge. All simulations for the mesh sensitivity study were conducted using the standard $k-\$$ turbulence model and canopy representation B2 as described in Section 3.4 .

Table 2. Details of the meshes used in the mesh sensitivity study.

\begin{tabular}{|c|c|c|c|c|c|}
\hline \multirow{2}{*}{ Mesh } & \multicolumn{2}{|c|}{ Maximum element size } & \multirow{3}{*}{$\frac{\text { Elements }}{2,600,616}$} & \multirow{3}{*}{$\frac{\text { Nodes }}{502,962}$} & \multirow{3}{*}{$\frac{\text { Time }}{29 \min }$} \\
\hline & Zone 1 & Zone 2 & & & \\
\hline Coarse & $15 \mathrm{~mm}$ & $20 \mathrm{~mm}$ & & & \\
\hline Medium & $7.5 \mathrm{~mm}$ & $10 \mathrm{~mm}$ & $16,774,974$ & $3,064,868$ & $167 \mathrm{~min}$ \\
\hline Fine & $5 \mathrm{~mm}$ & $7.5 \mathrm{~mm}$ & $33,601,429$ & $6,035,206$ & $727 \mathrm{~min}$ \\
\hline
\end{tabular}

In order to compare results for the Coarse, Medium and Fine meshes, values for 7 Tand $k$ were extracted from the converged solutions for a profile centred on the forest and located a distance of $2 \mathrm{H}_{c}$ in the lee of the obstruction. These profiles are presented in Fig. 9.

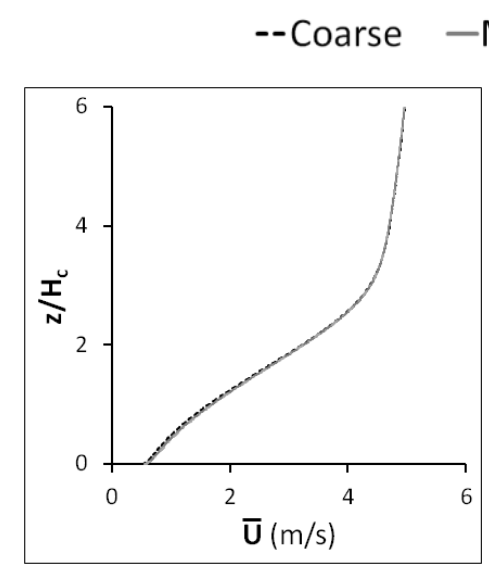

(a) Velocity -Medium - Fine

Fig. 9. Results of the mesh sensitivity study using the standard $k-\varepsilon$ turbulence model for a profile located at $2 H_{c}$ behind the forest.

As can be seen in Fig. $9(\mathrm{a})$, a minimal alteration to the simulated mean velocity values is obtained by refining the mesh. The effect of a finer resolution is observed to a greater degree in the turbulent kinetic energy profiles presented in Fig. 9 (b) where there is a difference in the magnitude of the 
peak simulated value of $0.06 \mathrm{~m}^{2} / \mathrm{s}^{2}$ between results achieved using the Coarse and Medium meshes. This reduces to a difference of just $0.02 \mathrm{~m}^{2} / \mathrm{s}^{2}$ when we compare values achieved using the Medium and Fine meshes which show strong agreement away from these peak values..

In order to set the findings of this mesh sensitivity study in context, we will also examine the quality of the three simulations using same methodology as for the results in the main body of this paper as presented in Section 4. To this end, data for both 7 Tand $k$ were extracted from each converged solution for the plane of investigation relevant to this work as described in Fig. 14. Values for the Normalised Percentage Error (NPE), defined in Section 4.1, were then calculated in order to compare results achieved using the Coarse and Fine meshes with those achieved using the Medium mesh. The results of these comparisons are presented as contour plots in Fig. 10. The scales used in Fig. 10 are identical to those used in Fig. 15 and Fig. 16 in order to set the results of the mesh sensitivity study in context.

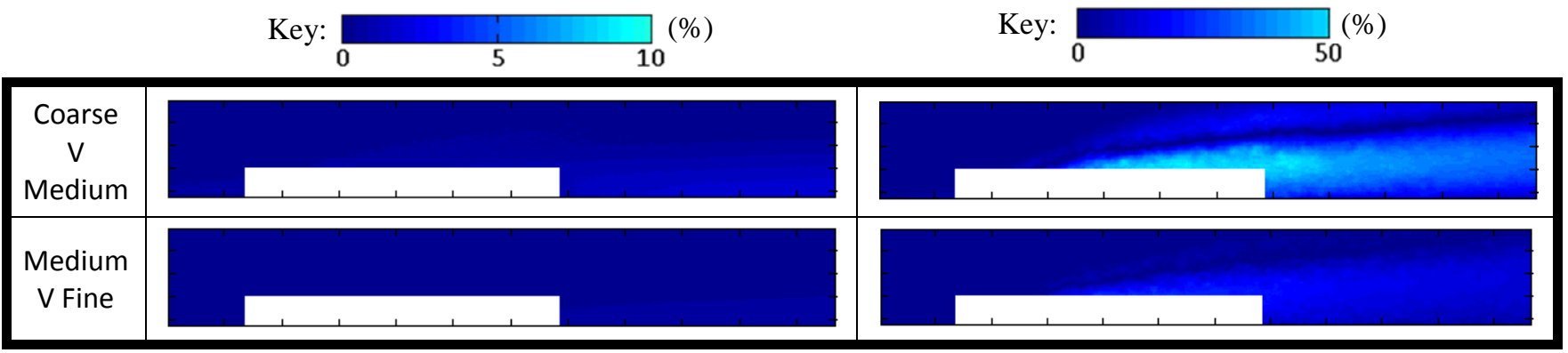

(a) Velocity

(b)Turbulent kinetic energy

Fig. 10. Results of the mesh sensitivity study using the standard $k-\varepsilon$ turbulence model for the plane of investigation as detailed in Fig. 14

A s can be seen from Fig. 10 the results achieved using the Fine mesh vary only very slightly from those achieved using the Medium mesh. This alteration is particularly pronounced in Fig. 10 (b) for the simulation of $k$, however, the magnitude of this discrepancy is considerably lower than the results presented for the main body of this paper in Fig. 15 and Fig. 16. Thus, we have opted to use the Medium mesh for all simulations as this arrangement provides a balance of accuracy and computational expense which is more applicable to the industrial flow calculations which this paper aims to influence.

The maximum observed skewness for the Medium mesh was 0.84 with a mean value of 0.2 and a standard deviation of 0.11. Generally, a skewness of $<0.95$ is taken as being acceptable and $<0.25$ deemed excellent (ANSYS Inc., personal communication, November, 2010).

\subsection{Representation of the canopy in the CFD model}

The effect of the forest canopy was simulated within the CFD model domain using varying levels of detail in order to ascertain the role that canopy morphology data can play in improving numerical simulations. These are described below:

B1 - The geometry of the canopy was modelled as a porous regular cuboid of $675 \mathrm{~mm} \times 675 \mathrm{~mm}$ and height equal to the average canopy height of $76 \mathrm{~mm}$, as shown in Fig. 11 . A constant $L A D=5.75 \mathrm{~m}^{-1}$ 
was applied throughout the cuboid. This value was obtained by averaging the values calculated using the method described in Section 2.5 in the $x$-streamwise, $y$-lateral and z-vertical dimensions. This homogenous description of forest canopies is commonly used both in industry and academic research (Lee, 2000 and Ross and Baker, 2013).

B2 - The canopy was again modelled as a porous regular cuboid, however, a greater level of canopy detail was introduced by allowing the leaf area density to vary in $z$ to reflect the actual porosity as shown in Fig. 6 . This is a subtle method of increasing canopy detail previously used by Wylie and Watson (2010) and Sogachev et al., (2009).

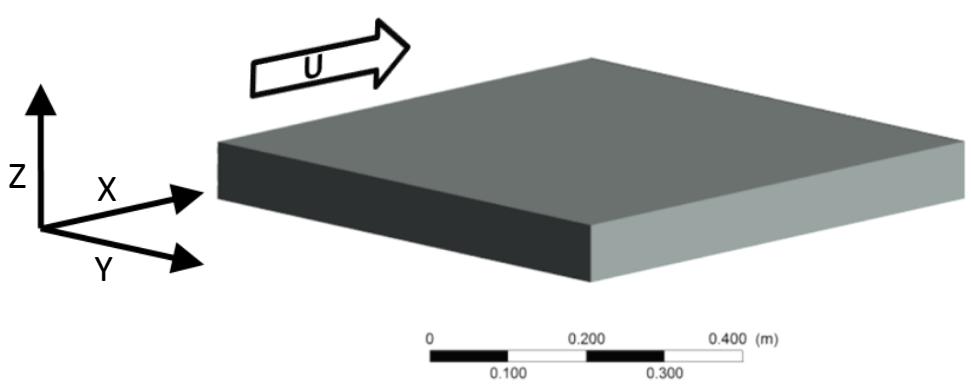

Fig. 11. Canopy geometry for B1 and B2.

The $\mathrm{U}$-arrow indicates the main component of the wind.

V1 - As the $x, y$ coordinates of each model tree were recorded during the experiments, it was possible to accurately model the variation in canopy height throughout the forest. This was achieved by introducing a surface into the CFD model domain which followed the $x, y, z$ coordinates of the highest point of each of the 100 trees. This then served as the top surface for a porous block representing the canopy, as shown in Fig. 12 . In this model the single value of $L A D=5.75 \mathrm{~m}^{-1}$ was used.

V2 - As V1, but the LAD profile was averaged in $x, y$ and allowed to vary in $z$ as shown in Fig.6.

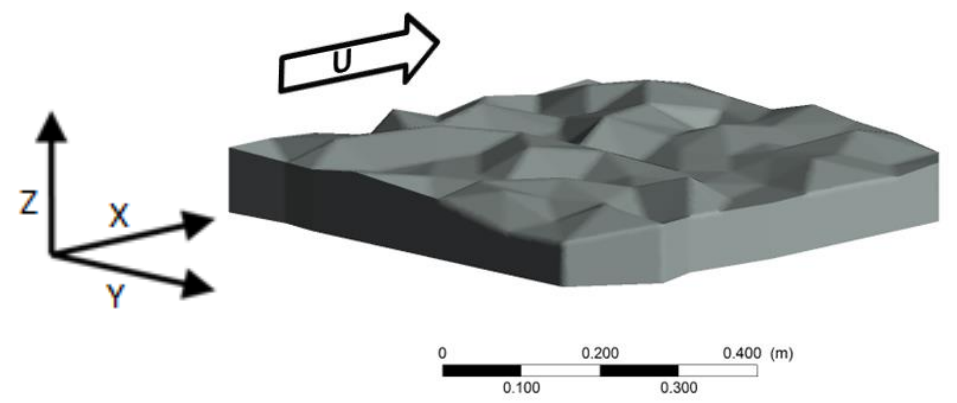

Fig. 12. Geometry used for V1 and V2.

The $U$-arrow indicates the main component of the wind. 
C1 - This model was inspired by the work of (Yue, 2007) who advocated the use of a plant scale approach when considering canopy flows. Each tree was modelled as an individual cylinder of height and diameter equal to the height and maximum diameter of the actual tree represented, as shown in Fig. 13. An average value of $L A D=32.5 \mathrm{~m}^{-1}$ was applied throughout each cylinder. This larger value is due to the fact that the volume used to calculate the $L A D$ is the annular volume of the individual tree rather than that of the entire forest.

C2 - The geometry was modelled as C1. An LAD was calculated for each tree using the appropriate annular volume. These profiles were then averaged. The resulting LAD profile follows the trend shown in Fig. 6 with the values for LAD increased, approximately by a factor of 6 .

C3 - Although the model forest is comprised of 100 unique model trees, there are, broadly speaking, ten categories. The trees were divided into these categories and a characteristic LAD profile was produced for each. The appropriate profile was then included in each cylindrical porous sub-domain.

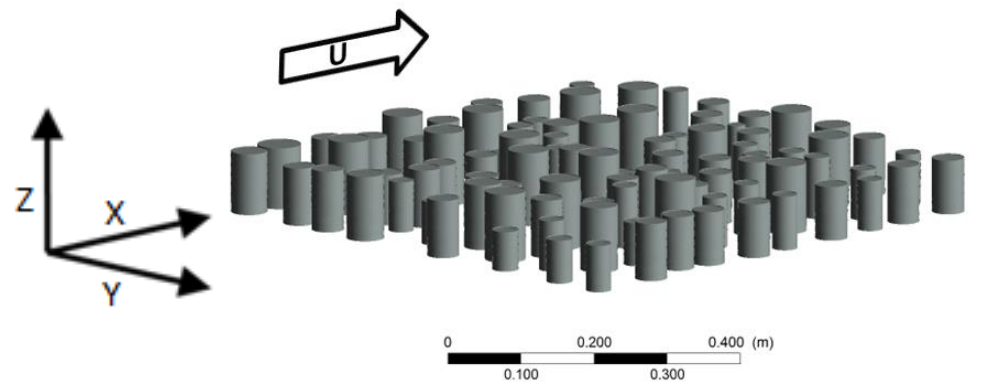

Fig. 13. Geometry used in C1, C2 and C3.

The $\mathrm{U}$-arrow indicates the main component of the wind.

The canopy representations used are summarised in Table 3.

Table 3. Summary of canopy representations used.

\begin{tabular}{|l|l|l|}
\hline Name & Geometry & LAD averaging \\
\hline B1 & Regular cuboid & $x, y, z$ \\
\hline B2 & Regular cuboid & $x, y$ \\
\hline V1 & Block with varying roof & $x, y, z$ \\
\hline V2 & Block with varying roof & $x, y$ \\
\hline C1 & $100 x$ Cylinders & $x, y, z$ \\
\hline C2 & $100 x$ Cylinders & $x, y$ \\
\hline C3 & $100 x$ Cylinders & By category \\
\hline
\end{tabular}

\section{Results}

\subsection{Quality metrics}

The stereo-PIV measurement technique used in the wind tunnel produces a large quantity of data for the plane investigated. In the present study, a total of 43,200 measurements on a $3.2 \mathrm{~mm} \times$ $3.2 \mathrm{~mm}$ grid were available covering the region shown in Fig. 14. Data were not available for less that $20 \mathrm{~mm}$ above the tunnel floor or canopy as reflectance from the surfaces interfered with data 
capture. It was also not possible to capture data during the experiments for approx $2 \mathrm{H}_{c}$ up and down-stream of the forest as the trees obscured the stereoscopic view. The stereo-PIV plane is centred on the forested area.

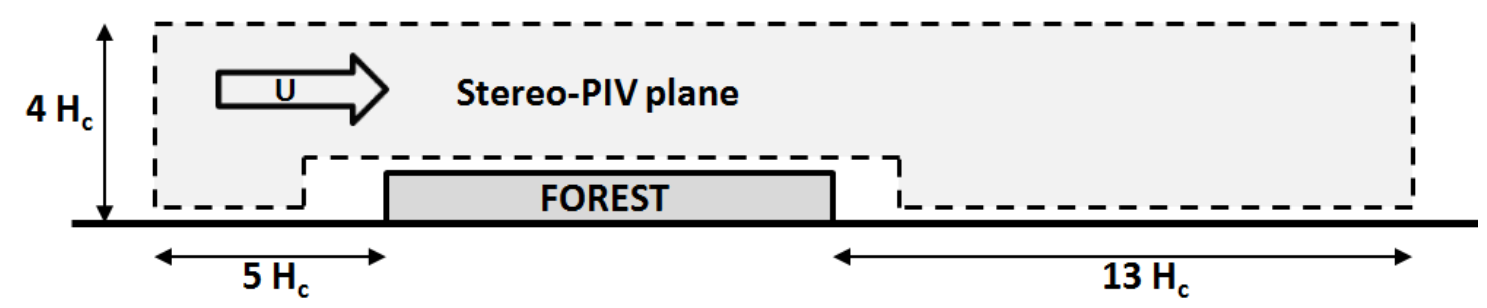

Fig. 14. Elevation showing extent of stereo-PIV measurement plane in relation to the model forest. The $\mathrm{U}$-arrow indicates the main component of the wind.

For each of the data points on the plane in Fig. 14, the modulus of the mean wind speed, 7 , was calculated as:

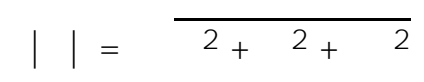

Where $\mathrm{T}^{\sharp}, t^{t}$ and $\mathrm{t}^{\sharp}$ in $\mathrm{m} / \mathrm{s}$ are the mean wind speeds in the $\mathrm{x}, \mathrm{y}$ and $\mathrm{z}$ directions, respectively. Values for $k$ were calculated as:

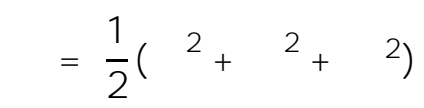

Where, $u^{\prime}, v^{\prime}$ and $w^{\prime}$ are the fluctuations in $\mathrm{m} / \mathrm{s}$ from the mean wind speeds $\mathrm{I}^{\prime \prime}, t^{+}$and $\mathrm{f}^{\prime \prime}$.

Values for $k$ and $T$, were also extracted from each CFD model run for all grid points in order to assess the accuracy of each simulation. The Pearson Correlation Coefficient, $R$, was calculated for both $k$ and ${ }^{*}$ Tfor each simulation using Eq. 15:

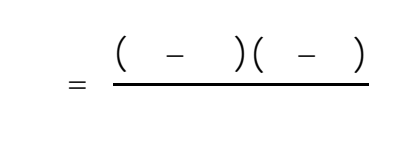

Where, $E$, refers to experimental values, $S$, refers to simulated, an over-bar denotes an average and $ढ$, the standard deviation. The values calculated for $R$ are generally high due to the large number of points which are contained in the free-stream where simulation errors are typically small. It would be possible to define a smaller region of more perturbed flow in which to calculate $R$, however, the definition of this region may bias results toward a particular simulation. A detailed discussion of this issue can be found in Holmes (2011).

Regardless of the high values obtained, $R$ gives a non-subjective qualitative assessment of the accuracy of the various CFD model simulations. In order to visualise where the error occurs for each 
simulation, contour plots are also provided which display the Normalised Percentage Error (NPE) for each of the grid points considered. The value of NPE for each point was calculated as:

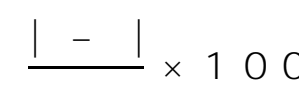

The reference, Ref, values used are $4.5 \mathrm{~m} / \mathrm{s}$ and $0.133 \mathrm{~m}^{2} / \mathrm{s}^{2}$ for 7 Tand $k$ respectively. Maximum NPE values of $25 \%$ and $150 \%$ are set for Tand $k$ in order to maintain a meaningful scale in the contour plots. Fig. 15 and Fig. 16 show contour plots for 7 tand $k$ respectively for both the standard k-\$ and SST turbulence closures for the extent of the stereo-PIV plane detailed in Fig. 14.

\subsection{Velocity}

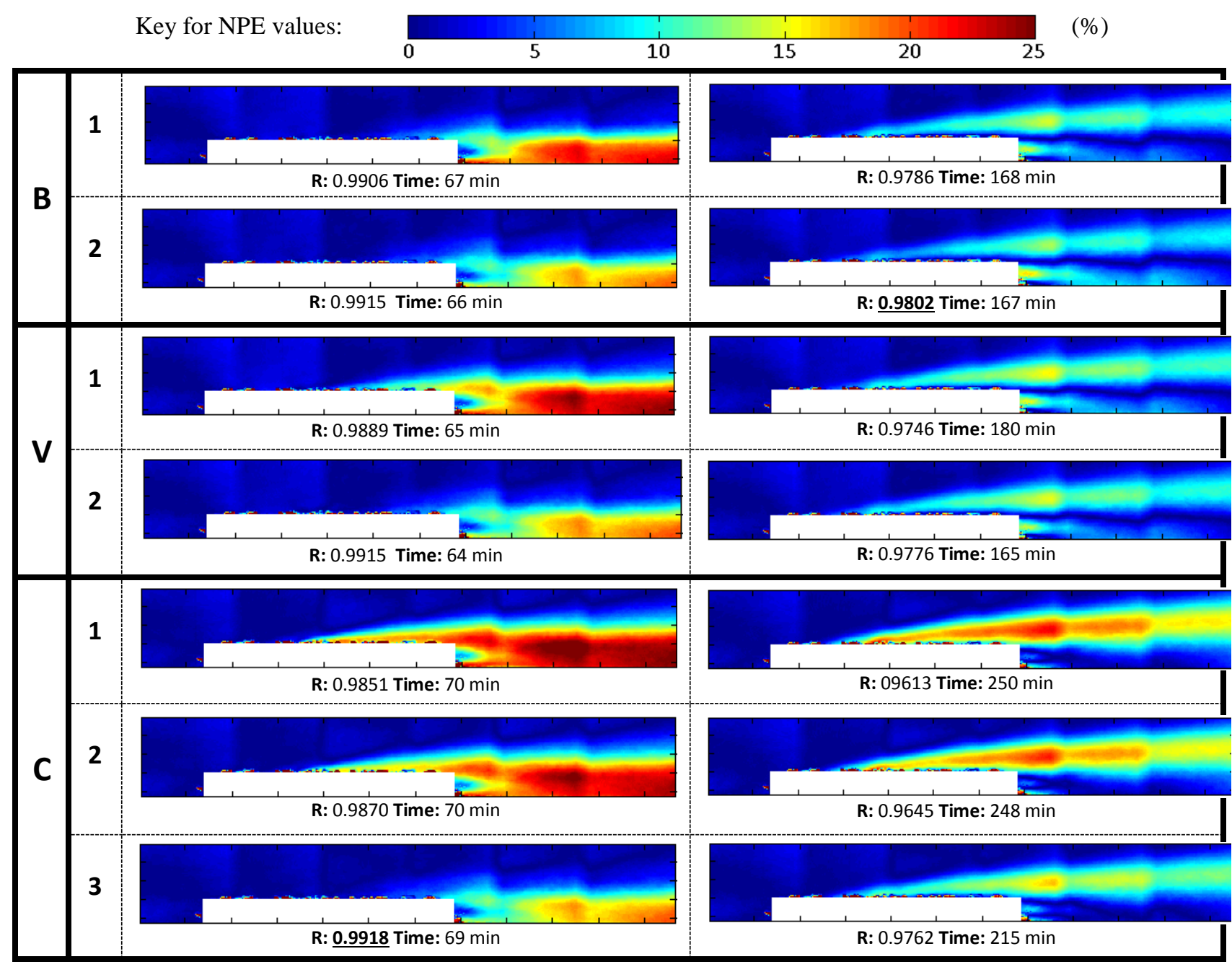

(a) SST

(b) $\mathrm{k}$ ₹

Fig. 15. These contour plots show the NPE between the simulations and the corresponding values from the wind tunnel. 
4.3 Turbulent kinetic energy

Key for NPE values:
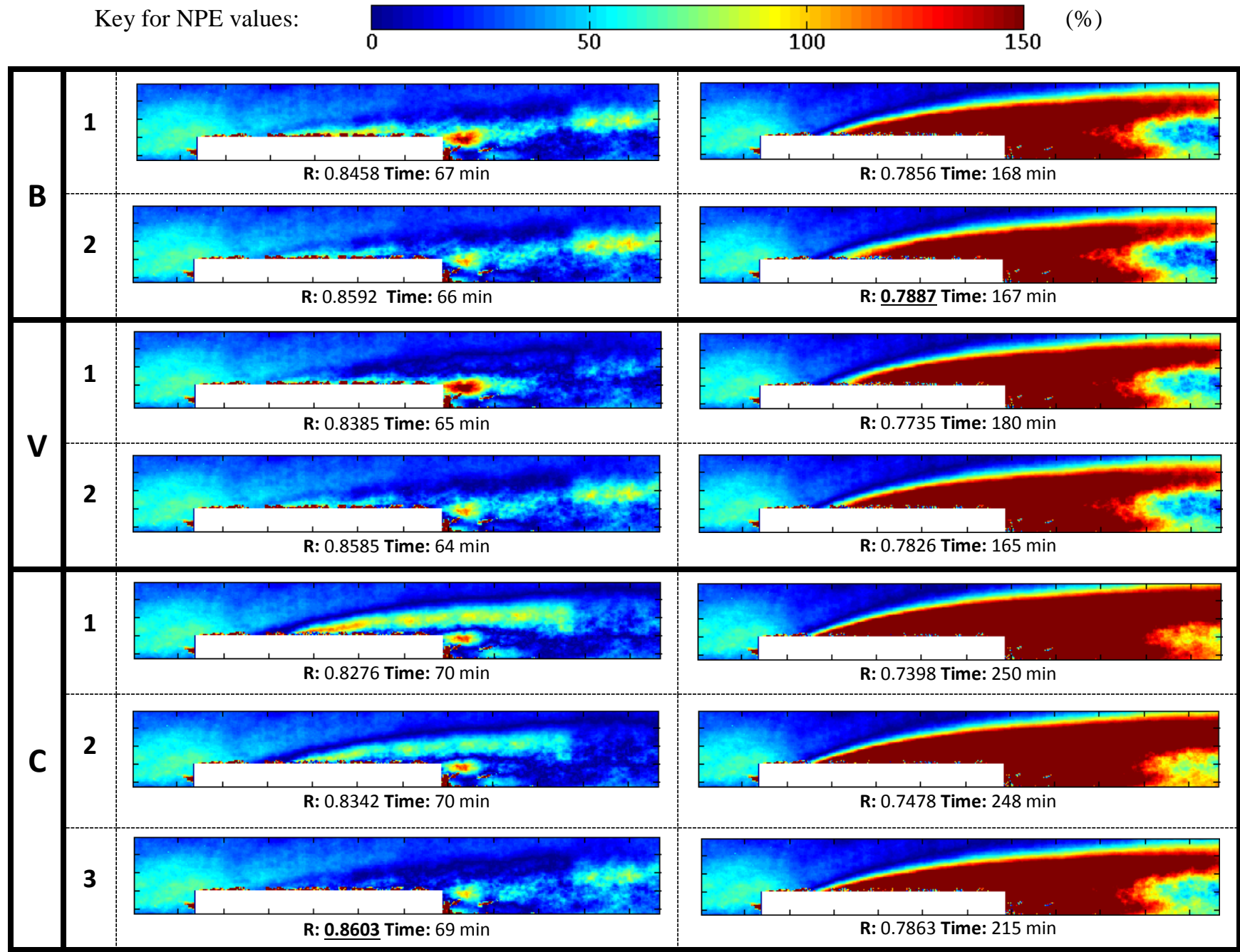

(a) SST

(b) $k-$ Y

Fig. 16. These contour plots show the NPE between the turbulent kinetic energy values calculated in the various CFD model simulations and the corresponding values from the wind tunnel. 


\section{Discussion}

As mentioned in Section 3.2, both inlet and local values were used for the $k$, №and $₹$ terms within Eqs. 10 -12. It was found that using local values resulted in slightly worse agreement with the wind tunnel measurements. This is most likely due to the feedback problem inherent in using local values as discussed in Section 3.2. Thus, all results shown in Fig. 15 and Fig. 16 were produced using inlet values for the $k$, №and ₹ terms within Eqs. $10-12$.

\subsection{Velocity}

Examining the contour plots for velocity in Fig. 15 alone would indicate that the standard k-\$ model produces less error. However, this appraisal is not borne out by examination of corresponding $R$ values for each canopy representation. This apparent contradiction is explained by Fig. 17, which shows the non-normalised Tprofile measured in the tunnel at a distance of $10 \mathrm{H}_{c}$ behind the canopy, compared with corresponding profiles simulated using both SST and standard k-\$ turbulence closures for the V1 canopy representation.

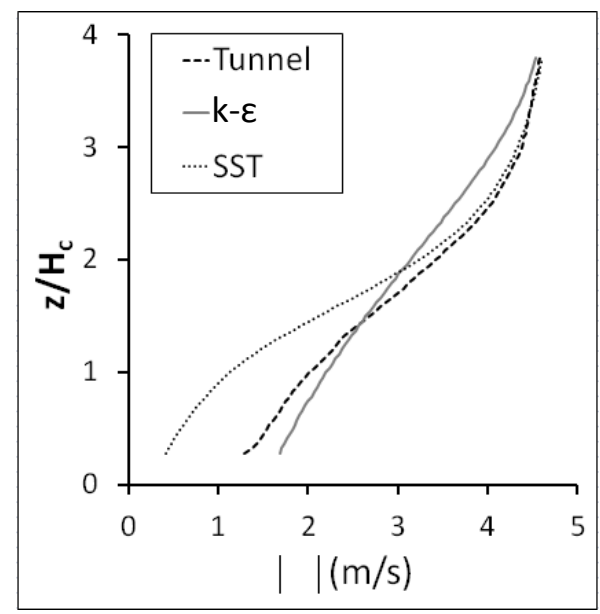

Fig. 17. Velocity profiles for SST and standard $k-l$ at $10 H_{c}$ for canopy representation V1 compared to tunnel measurements.

We see in Fig. 17 that the magnitude of the error between the modelled and simulated $X^{4}$ is larger for the SST turbulence model than for the standard $k$-\$ model for heights of less than $1.5 H_{c}$. However, the SST model gives a better approximation of the gradient of the actual velocity profile whilst the standard $k-\$$ model does not capture this detail. However, both models perform quite well as can be observed from the high values of $R$ and the fact that the error in the measurement campaign can be observed in the cyclical patterns in the contour plots which follow the positioning of adjacent stereo-PIV measurement planes.

No reduction in error is observed by introducing increasing levels of geometry data with contour plots for B2, V2 and C3 in Fig. 15 being very similar. However, there is a reduction in error achieved by increasing the level of LAD data. This is particularly true for the results obtained using the SST turbulence model where there is a considerable improvement between contour plots B1 and B2, V1 and $\mathrm{V} 2$, and $\mathrm{C} 1$ and $\mathrm{C} 3$ shown in Fig. 15(a). 


\subsection{Turbulent kinetic energy}

There is a much starker contrast observed between results produced using the SST turbulence model and the standard k-\$ model when we examine contour plots for $k$ shown in Fig. 16. It is clear that the SST model performs considerably better at simulating both the gradient and magnitude of the measured $k$ profiles. This is also seen in Fig. 18 , which shows the $k$ profile measured in the tunnel at a distance of $5 \mathrm{H}_{c}$ behind the canopy, compared with corresponding profiles simulated using both SST and standard $\mathrm{k}-\mathrm{\$}$ turbulence models for the $\mathrm{C} 3$ canopy representation.

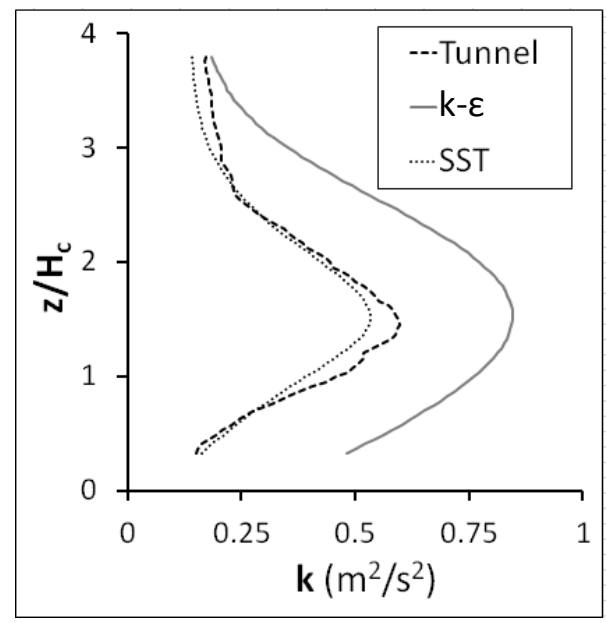

Fig. 18. $k$ profiles for SST and standard $k-\mathrm{l}$ at $5 H_{c}$ for canopy representation $\mathrm{C} 3$ compared to tunnel measurements.

As can be seen in Fig. 18, the SST model performs very well in capturing the magnitude of the turbulence which is often an area in which RANS simulations are poor. A significant proportion of the NPE in the highest quality simulation, i.e. C3 in Fig. 16(a), can in fact be attributed to the error due to the mismatch between the inlet $k$ profile used in the tunnel and the CFD model, as discussed in Section 3.3, rather than the simulation of the effect of the canopy.

Again, there is no observed reduction in error achieved by introducing increased levels of geometry data, with contour plots and $R$ values for $\mathrm{B} 2, \mathrm{~V} 2$ and $\mathrm{C} 3$ being very similar. However, a reduction in error is achieved by including increasing levels of LAD data. This trend can be observed in Fig. 16 (a) by comparing plots for $\mathrm{B} 1$ and $\mathrm{B} 2, \mathrm{~V} 1$ and $\mathrm{V} 2$, and $\mathrm{C} 1$ and $\mathrm{C} 3$. 


\subsection{Comparison to other canopy simulations}

There is a wealth of literature in which the flow around forests is investigated using numerical simulations, wind tunnel experiments and field studies. Through these investigations, authors have identified certain regions which are characteristic of canopy flows, an excellent review of which can be found in the literature survey presented in Lee (2000). In the following section, we will examine the observed flow field in the present study in order to ascertain if these characteristic regions are present. Thus, we will assess the applicability of the presented results to full scale canopy flows.

As illustrated by Fig. 14, the stereo-PIV plane is limited to a distance of $13 H_{c}$ behind the canopy and also does not cover regions close to surfaces. Thus, in order to carry out this comparison, we will examine results from the CFD model simulation which presents the least error. That is the simulation which used the C3 canopy representation along with the SST turbulence model. Elevation contour plots for this simulation are provided in Fig. 19 for both $k$ and $X$ The contour plots cover the entire CFD model domain and are on the same plane as the stereo-PIV measurements.

The characteristics of the various canopy flow regions are discussed in this section and their approximate extents in the current study are illustrated in Fig. 19. The discussion will again focus on aspects of the flow which are directly relevant to wind resource assessment.

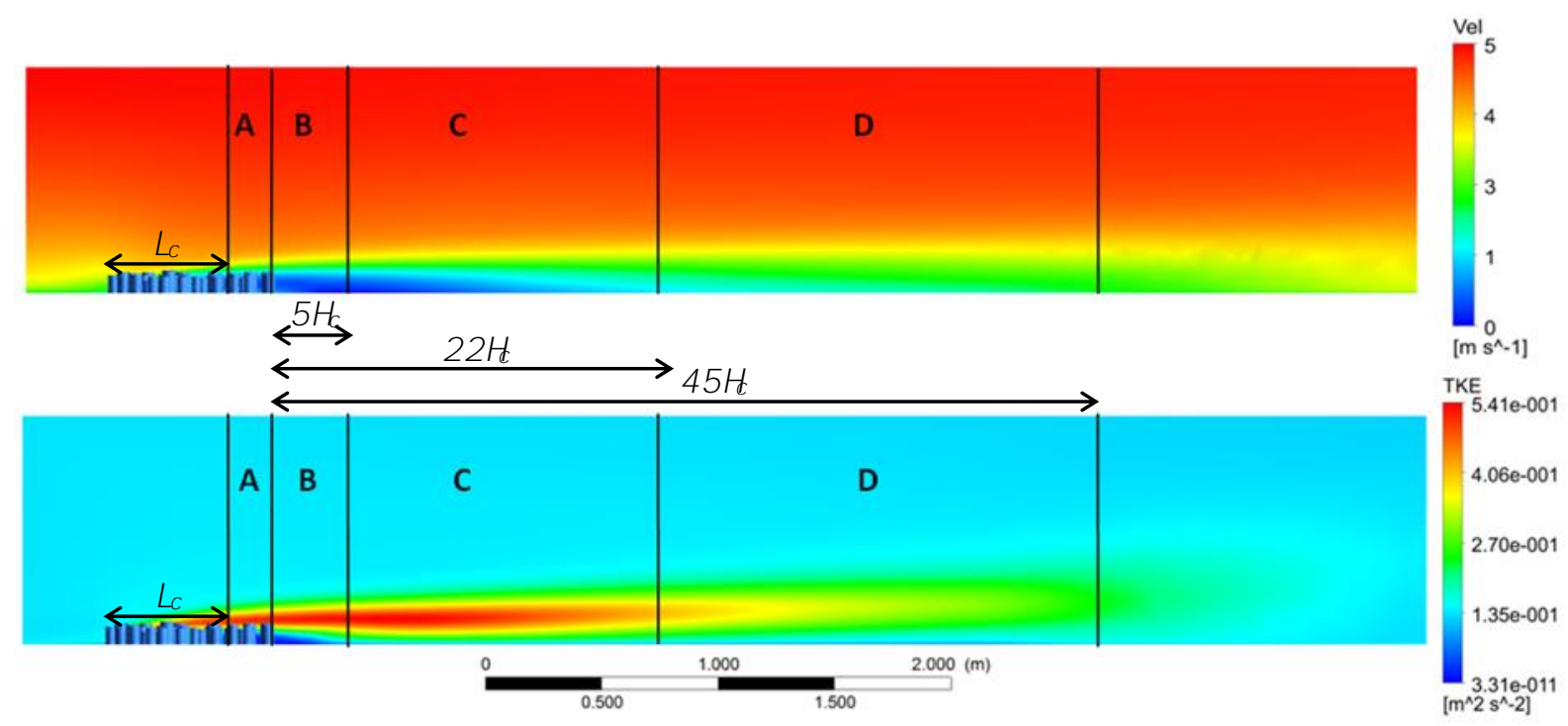

Fig. 19. Contour plots for $\mathrm{C}_{\mathrm{\Omega}}$ Tand $\mathrm{k}$ for the C3 canopy representation using the SST turbulence model. A - Developed canopy profile

In Section 2.4, we calculated the canopy adjustment length $L_{c}=479 \mathrm{~mm}$. In Fig. 19 we see that this is the approximate distance at which an internal boundary layer characteristic of the forest emerges. The subsequent region should thus be characteristic of a fully developed canopy flow. In order to investigate this flow region, a profile for mean horizontal wind speed is taken at a distance of $H_{c}$ from the leeward canopy edge. Velocity values are normalised to the mean horizontal wind speed above the canopy, $U_{H C}$, and the mean canopy height $H_{c}$.

The resulting normalised wind speed profile is shown in Fig. 20, where it is compared with characteristic developed canopy profiles as collated by Kaimal and Finnigan (1994). These 
characteristic profiles were measured in a number of wind tunnel and field studies examining canopy flows which are summarised in Table 4.

Table 4. Summary of data presented in Fig. 20.

\begin{tabular}{|l|l|l|}
\hline Name: & Canopy type & $\underline{\mathbf{H}}_{\mathbf{c}}(\mathrm{m})$ \\
\hline WT strips & Wind tunnel & .060 \\
\hline WT Wheat & Wind tunnel & .047 \\
\hline WT Rods & Wind tunnel & .190 \\
\hline Shaw Corn & Corn field & 2.6 \\
\hline Wilson Corn & Corn field & 2.25 \\
\hline Moga & Forest & 12 \\
\hline Uriarra & Forest & 20 \\
\hline Bordeaux & Forest & 13.5 \\
\hline
\end{tabular}

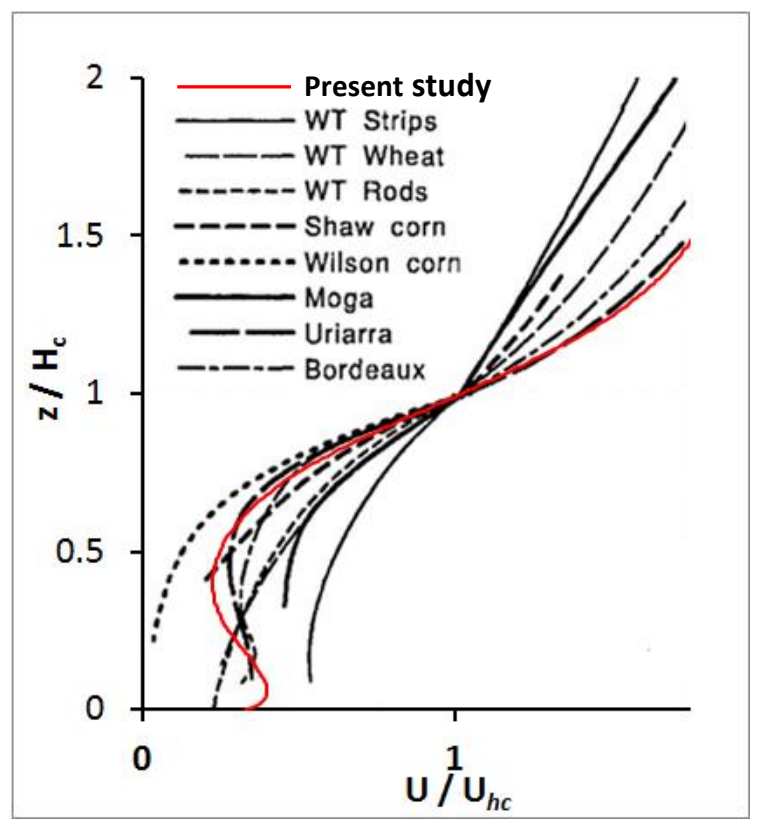

Fig. 20. Normalised flow profile in region A compared with profiles published in Kaimal and Finnigan (1994).

As can be seen in Fig. 20, there is good agreement between the fully developed canopy profile simulated in the present study and the characteristic profiles presented in the literature. The simulated profile follows measurements in the WT Wheat data particularly well, except for the speed up simulated close to the surface. This speed up, which is also observed in the Bordeaux data set, is referred to as the sub-canopy jet and has been observed in canopy studies as early as Shaw (1977).

The sub-canopy jet is expected in canopies with a sparse trunk space and is characterised by a sharp peak in velocity above the floor at the windward edge of the obstruction. This peak then reduces exponentially as the flow moves through the canopy. In the present study, this initial peak and subsequent decay is observed within the trunk space at a distance of approximately $5 \mathrm{~mm}$ above the tunnel floor. However, the jet is not fully eradicated before reaching the leeward canopy edge. This is most likely due to the sparse nature of the canopy, discussed in Section 2.4, which does not pose a sufficient blockage in the trunk space to oppose the flow. Energy cascades from the more 
significantly obstructed crown space and results in a sub-canopy jet which persists for $2 H_{c}$ behind the forest into flow region $B$.

B - Quiet zone

In this characteristic canopy flow region, the velocity profile is significantly affected by the presence of the canopy. The shelter effect results in a region of low speed flow beneath the canopy height and increased turbulence levels experienced at approximately $H_{c}$. This is a transition region where there is relatively little change as the flow has not yet begun to adjust to the new surface roughness.

Lee (2000) indicates that this region can persist for $4-7 H_{c}$. In the present study, a distance of $5 H_{c}$ can be estimated as seen in Fig. 19.

\section{C-Mixing zone}

The strong shear caused by the velocity deficit beneath the canopy height causes the region of turbulence at $H_{c}$ to increase in extent. Lee (2000) describes this as self destructive turbulence as it eradicates the very shear which is creating it by allowing energy to cascade from the free-stream and encourage the velocity to return to a logarithmic profile. This region is characterised by this increased mixing and the fact that the effect of the canopy is still present in the velocity profile.

D - Re-equilibrium zone

At this stage, the effect of the canopy on the velocity profile has been completely eradicated through mixing of the boundary layer and a logarithmic profile is once again observed. Lee (2000) gives a range of possible values at which this will occur. He points to a wind tunnel study (Chen et al., 1995) in which flow from a forest to a very flat field was simulated and the wake was found to persist to $22 H_{c}$. This value can be taken as an upper range, with Raynor (1971) approximating a value of $5 \mathrm{H}_{c}$ based on full-scale measurements of flow in the lee of a coniferous forest transitioning into an open field with a more realistic roughness length.

In the present study, a logarithmic profile was re-established at $22 H_{c}$, which is marked on Fig. 19 and is in line with the findings of (Chen et al., 1995). However, values of $k$ do not return to within $25 \%$ of ambient levels until approximately $45 H_{c}$. This distance is marked in Fig. 19 as the upper range of flow region $D$. This is a considerable distance which is influenced by the low ambient turbulence levels in the simulation due to the aerodynamic roughness length used.

In a recent experimental campaign presented in Lee and Lee (2012), the flow around a bank of three fir trees of $H_{c}=150 \mathrm{~mm}$ was investigated in a wind tunnel study using stereo-PIV. Although the presented blockage was far below the relevant canopy adjustment length, the effect of the wake on the velocity profile was found to persist until $10 \mathrm{H}_{c}$ in the lee of the blockage. In this context, the values of $22 \mathrm{H}_{c}$ and $50 \mathrm{H}_{c}$ for $\mathrm{k}$ T $\mathrm{T}$ and $\mathrm{k}$ respectively, as simulated by the CFD model, would not appear unreasonable. 


\section{Conclusions}

It was noted in Lopes da Costa (2007) and Wylie (2013) that the best turbulence model for predicting $k$ is not usually the best for predicting velocity when considering canopy flows. This is not the case for the present study where SST is found to consistently outperform the standard k-₹ turbulence closure model. The SST model is known to perform well when simulating adverse pressure gradients and separating flow (Menter, 1994) as it switches between k- $\varepsilon$ a twd e lq u a t i o n s d e p e n d i n g the proximity to roughness elements. It would appear that this ability has allowed the SST model to capture the turbulent fluctuations created by the extreme roughness which the canopy presents.

The greater computational expense of the standard $k$-\$ simulations indicates that this turbulence model struggled to resolve the turbulence generated by the canopy and this is further evidenced by the poor results presented in Fig. 16. This is unsurprising given the difficulties experienced with the $k$ ₹ model when considering flows near surface elements and lends further weight to the argument that this model should not be used when considering flows within the atmospheric boundary layer.

Despite the strong performance of the SST turbulence model in capturing the detail of the flow above and in the lee of the forest, a divergence between simulated and measured data is observed, even for the best simulations, near the floor in the final $4 H_{c}$ of the stereo-PIV measurement plane. This can be observed in Fig. 15(a) for the B2, V2 and C3 contour plots. By examining velocity profiles in this region it was found that the recovery of the flow predicted by the CFD simulation is slower than that observed in the tunnel. As the velocity profile is modelled accurately near the floor closer to the forest, this may indicate that the modelling constants used in the SST model require adjustment. However, given the cyclical nature of the error, which corresponds to the positioning of the stereo-PIV planes, it is possible that this divergence is due to experimental error.

This cyclic error in the experimental measurements can be dampened by further linearization of the data between successive stereo-PIV panes. However, this was deemed to be undesirable for the present study where the stated aim is to avoid unnecessary tuning. Also, despite this discrepancy, the improvement in CFD simulation quality by including additional morphology data is clearly observed in Fig. 15 and Fig. 16. This trend would not be significantly altered by removing the cyclic error from the experimental data.

In Section 5.3, the canopy flow produced by the forest of architectural model trees used for this study was compared with various other wind tunnel canopy models and field measurements. It was shown that the observed flow field and the recovery of the wake are similar to the characteristic canopy flows presented in the literature. This would indicate that the architectural model trees are a good candidate for wind tunnel experimentation of heterogeneous canopy flows. In addition, the use of the Tree Analyser software to capture the required LAD data allowed accurate simulation of the flow without the need to tune the CFD model. This is encouraging for cross-model validation and may allow other features of the flow field, such as the turbulent structures, to be investigated using LES and other unsteady CFD models.

Results indicate that the inclusion of accurate LAD profile data can significantly improve simulation quality without incurring additional computational expense. Unfortunately, the inclusion of realistic geometry data yields only minor improvements and, in the case of the standard $k-\varepsilon$ turbulence model, significant computational expense. However, it is important to note that accurate geometry 
data, such as tree height, diameter, location and forest footprint, were vital when deriving LAD profiles which precisely captured the variation of canopy density with height and, in the case of C3, in the three dimensions.

There may be some cause for concern in the interpretation of these results given the mismatch in the tunnel and CFD inlet turbulent kinetic energy profiles as shown in Fig. 8 (b). Whilst we wished to avoid any tuning of the turbulence models during this study, it is desirable to investigate the effect of removing this mismatch by adjusting the value of $C_{\mu}$. Thus, the simulations were also run using a $C_{\mu}$ value of 0.04 . It was found that whilst the quality of the simulations was generally improved, the observed trend, with regards the desirable level of canopy morphology and the relative performance of the SST and standard $k-\$$ model, remained the same.

From the results presented, it is clear that simulation quality of forested terrain can be improved by including data which captures the heterogeneous nature of forest canopies. These data can be derived using a combination of canopy geometry and LAD measurements. For robust numerical simulations for the purpose of wind resource assessment, it may be desirable to capture the seasonal, annual and forestry management variations associated with forests.

Fortunately, cheap and effective tools exist to effectively capture these morphology data, a review of which can be found in Jonckheere et al. (2004). These tools, which were developed for the forestry industry, would seem to provide a financially and computational inexpensive method of reducing uncertainty for the resource assessment industry.

Future work will address some of the shortcomings of the research presented in this paper. Specifically, additional stereo-PIV measurements will be taken in order to identify the point of flow reattachment in the lee of the canopy and assess the ability of the CFD models to predict this. Transient CFD runs will also be conducted using Large Eddy Simulation in order to investigate the turbulent structures generated by the canopy. In addition, there is scope to investigate the effect of atmospheric stability on canopy flows and the ability of CFD models to capture this detail by conducting stratified experiments in the EnFlo tunnel facility at the University of Surrey.

Finally, validation experiments using a similar methodology will be conducted using field data in order to assess if the results presented in this paper are valid at full scale.

\section{Acknowledgments}

This work has been carried out with funding from the EU FP7-PEOPLE program under WAUDIT Marie-Curie Initial Training Network.

\section{References}

Andersen, H., McGaughey, R. J., Reutebuch, S. E., 2005. Estimating forest canopy fuel parameters using LIDAR data. Remote Sensing of Environment 94, 441-449.

Aubrun, S., Leitl, B., 2004. Development of an improved physical modelling of a forest area in a wind tunnel. Atmospheric Environment 38, 2797-2801.

Bai, K. Meneveau, C., Katz, J., 2012. Near wake turbulent flow structure and mixing length downstream of a fractal tree. Boundary Layer Meteorology 143, 285-308. 
Bechmann, A., Sørensen, N. N., Berg, J., Mann, J., Réthoré, P. E., 2011. The Bolund Experiment, Part II: Blind Comparison of Microscale Flow Models. Boundary Layer Meteorology 141, 245-271

Belcher, S.E., Jerram, N., Hunt, J.C.R., 2003. Adjustment of a turbulent boundary layer to a canopy of roughness elements. Journal of Fluid Mechanics 488, 369-398.

Belcher, S.E., Harman, I.N., Finnigan, J.J., 2012. The Wind in the Willows. Flows in forest canopies in complex terrain. Annual review of Fluid Mechanics 44, 479-504.

Bohm, M., Finnigan, J.J., Raupach, M.R. , 2013. Turbulent structure within and above a canopy of bluff elements. Boundary Layer Meteorology 146, 393-419.

Burlando, M., Georgieva, E., Ratto, C.F., 2007. Parametrisation of the PBL for diagnostic wind models. Boundary Layer Meteorology 125, 389-397.

Burns, S.P., Sun, S., Lenschow, D.H., Oncley, S.P., Stephens, B.B., Yi, C., Anderson, D.E., Hu, J., Monson, R.K., 2011. Atmospheric stability effects on wind fields and scalar mixing within and just above a subalpine forest in sloping terrain. Boundary Layer Meteorology 138, 231-262.

Chen, J.-M., Black, T.A., Novak, M.D., Adams, R.S., 1995. A wind tunnel study of turbulent airflow in forest clear cuts. In: Coutts, M.P., Grace, J. (Eds.), Wind and Trees. Cambridge University Press, 7187.

Desmond, C.J., Watson, S.J., Wind flow around a single tree: A study on the sensitivity of CFD models. in: proceedings of ICWE-13, 2011.

Desmond, C.J., Watson, S.J., Sayer, A., Hancock, P., Forest canopy flows in non-neutral stability. in: Proceedings of EWEA, 2012.

Dupont, S., Gosselin, F., Py, C., De Langre, E., Hemon, P., Brunet, Y., 2010. Modelling waving crops using LES. Comparison with experiments and a linear stability analysis. Journal of Fluid Mechanics $652,005-44$.

Dupont, S., Irvine, M.R., Bonnefond, J., Lamaud, E., Brunet, Y., 2012. Turbulent structures in a pine forest with a deep and sparce trunk space. Boundary Layer Meteorology 143, 309-336.

Endalew, A.M., Hertog, M., Delele, M.A., Baetens, K., Persoons, T., Baelmans, M., Ramon, H., 2009 a. CFD modelling and wind tunnel validation of airflow through plant canopies using 3D canopy architecture. International Journal of Heat and Fluid Flow 30, 356-368.

Endalew, A.M., Hertog, M., Gebrehiwot, G.G., Baelmans, M., Ramon, H., Nicolaï, B.M., Verboven, P., 2009 b. Modeling airflow within model plant canopies using an integrated approach. Computers and Electronics in Agriculture 66, 009-24.

Finnigan, J.J., 2010. Waving plants and turbulence. Journal of Fluid Mechanics 652, 001-004.

Franke, J., Hellsten, A., Schlünzen, H. and Carissimo, B., Best Practise Guideline for the CFD Simulation of Flows in the Urban Environment. COST Office 
Holmes, H.A., Quality assurance of wind energy assessment models. Deliverable 24, WAUDIT FP7 contract No. 238576, 2011.

Jonckheere, I., Fleck, S., Nackaerts, K., Muys, B., Coppin, P., Weiss, M., Baret, F., 2004. Review of methods for in situ LAI determination. Agricultural and Forest Meteorology 121, 19-35.

Juretić, F., Kozmar, H., 2013. Computational modeling of the neutrally stratified atmospheric boundary layer flow using the standard $k-\varepsilon$ turbulence model. Journal of Wind Engineering and Industrial Aerodynamics 115, 112-120.

Kaimal, J.C., Finnigan, J.J., Atmospheric boundary layer flows. Oxford University Press, 1994.

Launder, B.E., Spalding D.E., Mathematical models of turbulence. Academic Press, 1972.

Lee, J.P., Lee, S.J., 2012. PIV analysis on the shelter effect of a bank of real fir trees. Journal of Wind Engineering and Industrial Aerodynamics 110, 40-49.

Lee, X., 2000. Air motion within and above forest vegetation in non ideal conditions. Forest Ecology and Managment 135, 003-18.

Liang, L., Xiaofeng, L., Borong, L., Yingxin, Z., 2005. Improved k- $\varepsilon \quad$ t wo e q u a t i o n t u r b u l e canopy flows. Atmospheric Environment 40, 762-770.

Liu, J., Chen, J.M., Black, T.A., Novak, M.D., 1998. E- $\varepsilon$ mo d e I I i n g lowfdownwinid df al I e $n$ t a i model forest edge. Boundary-Layer Meteorology 72, 21-44.

Lopes da Costa, J.C., Atmospheric Flow over Forested and Non-forested Complex Terrain. PhD thesis, University of Porto, 2007.

McCormick, S., Montavon, C., Jones, I., Staples, C., Sinai, Y., Atmospheric boundary layer - Boundary layer conditions and profiles. WindModeller Manuals. ANSYS, 2012.

Menter, F.R., 1994. Two equation eddy viscosity turbulence models for engineering applications. AIAA Journal 32, No. 8, 1598-1605.

Meroney, R.N., 1968. Characteristics of wind and turbulence in and above forests. Applied Meteorology 7. No. 5, 780-788.

Molina-Aiz, F.D., Valera, D.L., Àlvarez, A.J., Madueño, A., 2006. A wind tunnel study of airflow through horicultural crops. Determination of the drag coefficient. Biosystems Engineering 93. No. 4, 447-457.

Omasa, K., Hosoi, F., Konishi, A., 2006. 3D lidar imaging for detecting and understanding plant responses and canopy structure. Journal of Experimental Botany 58, 881-898.

Petersen, G., Wind tunnel modelling of atmospheric boundary layer flow over hills. PhD. thesis, University of Hamburg, 2013.

Raynor, G.S., 1971. Wind and temperature structure in a coniferous forest and a contiguous field. Forensic Science 17, 351-363. 
Richards, P.J., Hoxey, R.P., 1993. Appropiate boundary conditions for computational wind engineering models using the k-epsilon turbulence model. Journal of Wind Engineering and Industrial Aerodynamics 46, 145-153.

Rodrigo, J.S., van Beeck, J., Dezsö -Weidinger, G., 2007. Wind tunnel simulations of the wind conditions inside bidimensional forest clear cuts Application to wind turbine siting. Journal of Wind Engineering and Industrial Aerodynamics 95. No. 7, 609-634.

Ross, A.N., Baker, T.P., 2013. Flow over partially forested ridges. Boundary Layer Meteorology 146, 375-392.

Ruck, B., Frank, C., Tischmacher, M., 2012. On the influence of windward edge structure and stand density on the flow characteristics at forest edges. European Journal of Forest Research 131, 177 189.

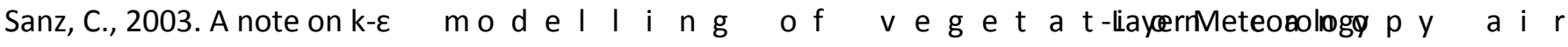
108, 191-197.

Schröder, A., Willert, C.E., Particle image velocimetry. New developments and recent applications. Springer, 2008.

Seidel, D., Fleck, S., Leuschner, C., 2012. Analyzing forest canopies with ground based laser scanning. A comparison with hemispherical photography. Agricultural and Forest Meteorology 154-155, 0018.

Shaw, R.H., 1977. Secondary wind speed maxima inside plant canopies. Journal of Applied Meteorology 16, 514-523.

Sogachev, A., Wind energy availability above gaps in a forest. In: Proceedings of EWEC , 2009.

Sogachev, A., 2009. A note on two equation closure modelling of canopy flow. Boundary Layer Meteorology 130, 423-435.

Sogachev, A., Kelly, M., Leclerc, M.Y., 2012. Consistent two equation modelling for atmospheric research. Buoyancy and vegetation implementations. Boundary Layer Meteorology 145. No. 2, 307327.

Stacey, G.R., Belcher, R.E., Gardiner, B.A., 1994. Wind flows and forces in a model spruce forest. Boundary Layer Meteorology 69. No. 3, 311-334.

Svensson,U., Haggkvist. H., 1990. A Two-Equation Turbulence Model for Canopy Flows. Journal of Wind Engineering and Industrial Aerodynamics, 35, 201-211

VDI - guildeline 3783/12, Physical modelling of flow and dispersion processes in the atmospheric boundary layer- application of wind tunnels. Beuth Verlag, 2000.

Wilcox, D.C., Turbulence modelling for CFD. DCW Industries,1993.

Wylie, S.J., CFD modelling of wind flow through and around forest canopies, PhD. thesis, Loughborough University, 2013. 
Wylie, S.J., Watson, S.J., Comparison of different turbulence closure schemes used for CFD modelling of wind flow around a forest simulated in a wind tunnel. in: Proceedings of EWEC, 2010

Yang, B., Raupach, M.R., Shaw, R.H., Kyaw Tha Paw U, Morse, A.P., 2006. LES of turbulent flow across a forest edge. Boundary Layer Meteorology 120, 377-412.

Yue, W., Parlange, M.B., Meneveau, C., Zhu, W., van Hout, R., Katz, J., 2007. Large eddy simulations of plant canopy flows using plant scale representation. Boundary Layer Meteorology 124, 183-203.

Zhang, M., Shen, G., Wei, R., Error analysis of stereoscopic particle image velocimetry. in:

Proceedings of SPIE 5058, Optical Technology and Image Processing for Fluids and Solids Diagnostics, 2002. 
